\title{
Unitary Representations of the Group of Diffeomorphisms via Restricted Product Measures with Infinite Mass II
}

\author{
by \\ Hiroaki SHIMOMURA
}

\begin{abstract}
This paper concerns the problem of irreducibly decomposing unitary representations of the group $\operatorname{Diff}_{0}(M)$ of diffeomorphisms with compact support on the smooth manifold $M$. As was shown in [19], these representations are decomposable under a fairly mild condition. In this paper, we consider a specific example of unitary representations $\left(T, \operatorname{Diff}_{0}(M)\right)$ that has been considered by [4]. $\left(T, \operatorname{Diff}_{0}(M)\right)$ is already a factor representation of type $\mathrm{II}_{\infty}$; in addition, it may be decomposed into irreducible components through the left regular representation of the group $\mathfrak{S}_{\infty}$ of finite permutations. We describe concrete realization of these irreducible components. The results obtained herein bear some resemblance to the finite-dimensional case of [20] with the exception of the factor representation. In addition in the Appendix, we will give another proof of the irreducibility and equivalence that were obtained in [4].
\end{abstract}

2010 Mathematics Subject Classification: Primary 58D05; Secondary 22E65.

Keywords: group of diffeomorphisms, unitary representation, irreducible component, restricted product measure.

\section{$\S 1$. Introduction}

Let $M=M^{d}$ be a paracompact $C^{\infty}$ manifold, and let $\operatorname{Diff}_{0}(M)$ be the group of all $C^{\infty}$ diffeomorphisms with compact support on $M$ with the natural topology $\tau$. Various authors have studied and constructed many interesting unitary representations of $\left(\operatorname{Diff}_{0}(M), \tau\right)$, as well as their linear versions, most of which are irreducible. However, as far as we know, the important problem of whether it is possible to decompose a given unitary representation of an infinite-dimensional group into irreducible ones has not been considered.

Communicated by S. Mukai. Received April 6, 2006. Revised July 18, 2006 and May 31, 2011.

H. Shimomura: 3-10-5 Den-enchofu, Ota, Tokyo 145-0071 Japan;

e-mail: DQE00332@nifty.ne.jp

(C) 2012 Research Institute for Mathematical Sciences, Kyoto University. All rights reserved. 
So we have recently investigated the above problem in the context of the group $\operatorname{Diff}_{0}(M)$, and of more general groups, including some infinite-dimensional groups [19]. In brief, this previous work may be summarized as follows: As is well-known, $\operatorname{Diff}_{0}(M)$ is a nuclear group, or an inductive limit of such groups according as $M$ is compact or non-compact. Therefore, we expected to find infinite-dimensional versions of the results on irreducible decompositions of unitary representations of locally compact groups due to Mautner [8, 9]. After checking his results carefully, we found that the existence of (finite) measures with quasi-invariance under dense translations was crucial (for example, right shifts, like the Shavgulidze measure $[15,17]$ on the group of diffeomorphisms).

We first worked with pairs of groups, $(G, H), H \subseteq G$, which have a probability measure $\mu$ on $G$ that is right $H$-quasi-invariant, and studied the irreducible decompositions of unitary representations of $G$ restricted to $H$. We then proceeded to the inductive limit. Of course, we were interested in the case that $\left(H, \tau_{H}\right)$ is dense in $\left(G, \tau_{G}\right)$. We showed that the abstract form of the decomposition problem has an affirmative answer.

Next, we considered the group $\operatorname{Diff}_{0}(M)$, where $M$ is compact, and showed that the decomposition problem has an affirmative answer under the fairly mild condition that a given unitary representation $T(\phi), \phi \in \operatorname{Diff}_{0}(M)$, is continuous with respect to the topology of uniform convergence of the maps $\phi$, together with their derivatives of order less than or equal to some $k$. As mentioned above, the Shavgulidze measure played a crucial role in these arguments. Applying inductive limit methods, we arrived at a similar result in the non-compact case. This is the summary of the previous paper [19].

In contrast, the present paper concerns unitary representations of $\operatorname{Diff}_{0}(M)$ which have already been considered in $[4,18]$; however, realizations of their irreducible decompositions were left for further work. We now provide a concrete description through the decompositions of the left regular representation of the finite permutation group $\mathfrak{S}_{\infty}$. We first recall the notation used not in [4] but in [18] because it is convenient to present a new result: Let $M$ be a connected, noncompact but $\sigma$-compact, smooth manifold with $d:=\operatorname{dim}(M) \geq 3$, let $\operatorname{Diff}_{0}(M)$ be the group of all smooth diffeomorphisms on $M$ with compact support, and $\mu$ be a smooth locally Euclidean measure on $M$ with infinite mass. Take a restricted product measure $\nu_{E}$ of countably many copies of $\mu$ depending on a family $E=\left\{E_{n}\right\}_{n}$ of disjoint Borel sets in $M$ which satisfies $0<\mu\left(E_{n}\right)<\infty$ and $\sum_{n=1}^{\infty}\left|1-\mu\left(E_{n}\right)\right|<\infty$ as in the context of Moore [10] (the details will be given in the next section). $\nu_{E}$ is quasi-invariant under the diagonal action of $\operatorname{Diff}_{0}(M)$. It follows that we have a natural representation $T(\phi)$ of $\phi \in \operatorname{Diff}_{0}(M)$ on the representation Hilbert space 
$\mathrm{L}_{\nu_{E}}^{2}\left(M^{\infty}\right)$ (the formulations used here are an extension of, and variation on the work described in [20] on finite direct product spaces). Notice that $\nu_{E}$ is invariant under finite permutations of coordinates, and the group $\mathfrak{S}_{\infty}$ acts on $\mathrm{L}_{\nu_{E}}^{2}\left(M^{\infty}\right)$ as another unitary representation $R(\sigma), \sigma \in \mathfrak{S}_{\infty}$.

Note that, as we show in Section 3, the representation $T(\phi) R(\sigma)$ of $\operatorname{Diff}_{0}(M) \times$ $\mathfrak{S}_{\infty}$ is irreducible. In other words, $\left(T, \mathrm{~L}_{\nu_{E}}^{2}\left(M^{\infty}\right)\right)$ and $\left(R, \mathrm{~L}_{\nu_{E}}^{2}\left(M^{\infty}\right)\right)$ are factor representations of type $\mathrm{II}_{\infty}$ and of type $\mathrm{II}_{1}$, respectively. It is interesting that these representations have different characteristics when compared with the natural representations described by [20] on the finite-dimensional space $M^{n}, n \in \mathbb{N}$.

Given $\mu, E=\left\{E_{n}\right\}_{n}$ and an irreducible unitary representation $(\Pi, H)$ of $\mathfrak{S}_{\infty}$, consider the Hilbert space $\mathcal{H}(\Sigma), \Sigma:=(\mu, E, \Pi)$, of all Borel measurable $H$-valued functions $f$ on $M^{\infty}$ such that

$$
f(x \sigma)=\Pi(\sigma)^{-1} f(x) \quad \text { for all } \sigma \in \mathfrak{S}_{\infty},
$$

and

$$
\|f\|_{E}^{2}:=\int_{D_{E}}\|f(x)\|_{H}^{2} \nu_{E}(d x)<\infty,
$$

where $D_{E}$ is a Borel set such that $D_{E} \sigma \cap D_{E}=\emptyset$ if $\sigma \neq \mathrm{id}$, and the complement of $\bigcup_{\sigma \in \mathfrak{S}_{\infty}} D_{E} \sigma$ is $\nu_{E}$-null, where $D_{E} \sigma:=\left\{x \sigma \mid x \in D_{E}\right\}$.

It is easy to see that $\operatorname{Diff}_{0}(M)$ acts on the Hilbert space $\mathcal{H}(\Sigma)$, and hence we have its natural representations that are all irreducible by [4]. As the last fact is fundamental, we will give another proof of it in the Appendix. What was left for further work in [4] is the question of whether these are actually irreducible components of the natural representation $\left(T, \mathrm{~L}_{\nu_{E}}^{2}\left(M^{\infty}\right)\right)$. We are now in a position to prove that this is the case (the details will be seen in the final section), and the successive steps of this proof are as follows:

$$
\begin{aligned}
\mathrm{L}_{\nu_{E}}^{2}\left(M^{\infty}\right) & \simeq \ell^{2}\left(\mathfrak{S}_{\infty}\right) \otimes \mathrm{L}_{\nu_{E}}^{2}\left(D_{E}\right) \\
& \simeq \int^{\oplus} H_{\lambda} \otimes \mathrm{L}_{\nu_{E}}^{2}\left(D_{E}\right) \sqrt{d \sigma(\lambda)} \\
& \simeq \int^{\oplus} \mathrm{L}_{\nu_{E}}^{2}\left(D_{E}, H_{\lambda}\right) \sqrt{d \sigma(\lambda)} \\
& \simeq \int^{\oplus} \mathcal{H}\left(\Sigma_{\lambda}\right) \sqrt{d \sigma(\lambda)} .
\end{aligned}
$$

The first line is due to the choice of the set $D_{E}$, the second line is a consequence of an irreducible decomposition of the left regular representation $L$ of $\mathfrak{S}_{\infty}$ with spectral measure $\sigma$, and the fourth line is derived from the third line by the natural map with $\Sigma_{\lambda}:=\left(\mu, E, \Pi_{\lambda}\right)$, where $\Pi_{\lambda}$ is an irreducible component of $L$. Notice that the corresponding maps at each stage have no connections with the representations 
of $\operatorname{Diff}_{0}(M)$, but that the composition of all of the maps is an intertwining unitary operator of their natural representations.

Throughout, we assume that $M$ is connected, $\sigma$-compact with $\operatorname{dim}(M) \geq 3$, but not compact; we must also impose a fairly mild condition (mcc) (stated just before Theorem 2.3) on $M$, for the technical reasons explained in the previous paper [18]. One might try proving the same results without (mcc), but this would most probably require much longer arguments.

As a rule, we follow the notation and terminology used by [18], and we recall these briefly in the next section.

\section{$\S 2$. Notation and basic arguments}

\section{$\S 2.1$. Restricted product measure with infinite mass}

We begin by introducing the notion of restricted product measure. Suppose that we are given, for each $n$, a $\sigma$-finite measure space $\left(X_{n}, \mathfrak{B}_{n}, \mu_{n}\right)$ and a set $E_{n} \in \mathfrak{B}_{n}$ with $0<\mu_{n}\left(E_{n}\right)<\infty$. Denoting the restriction of $\mu_{n}$ to $E_{n}$ by $\mu_{n} \mid E_{n}$, we form the product measure

$$
\hat{\nu}_{n}:=\frac{\mu_{1} \times \cdots \times \mu_{n}}{\mu_{1}\left(E_{1}\right) \cdots \mu_{n}\left(E_{n}\right)} \times \prod_{k=n+1}^{\infty} \frac{\mu_{k} \mid E_{k}}{\mu_{k}\left(E_{k}\right)} .
$$

As $\hat{\nu}_{n}$ is increasing in $n$, we then obtain a $\sigma$-finite measure $\hat{\nu}_{E}:=\lim _{n \rightarrow \infty} \hat{\nu}_{n}$ on the product measurable space $\left(X^{\infty}, \mathfrak{B}^{\infty}\right)$ of $\left(X_{n}, \mathfrak{B}_{n}\right)$.

In what follows, all the measure spaces $\left(X_{n}, \mathfrak{B}_{n}, \mu_{n}\right)$ are identical: $(M, \mathfrak{B}(M), \mu)$, where $M$ is a connected, non-compact but $\sigma$-compact, manifold of class $C^{\infty}, \mathfrak{B}(M)$ is the Borel field and $\mu$ is a smooth, locally Euclidean measure whose total mass is infinite.

Definition 2.1 (Unital sequence). A sequence $E=\left\{E_{n}\right\}_{n}$ of Borel sets in $M$ is said to be $\mu$-unital if it satisfies the following two conditions:

(1) for all $n, 0<\mu\left(E_{n}\right)<\infty$,

(2) $\sum_{n=1}^{\infty}\left|1-\mu\left(E_{n}\right)\right|<\infty$.

In addition, if the $E_{n}$ are mutually disjoint, we call it a disjoint $\mu$-unital sequence.

Without confusion, let us denote the product $\prod_{n=1}^{\infty} E_{n}$, which is called a unital product set, by the same letter $E$.

Definition 2.2 (Cofinality). Two $\mu$-unital sequences, $E=\left\{E_{n}\right\}_{n}$ and $F=\left\{F_{n}\right\}_{n}$, are said to be cofinal, written $E \sim F$, if they satisfy $\sum_{n=1}^{\infty} \mu\left(E_{n} \ominus F_{n}\right)<\infty$. 
In addition, if $E_{n}=F_{n}$ for sufficiently large $n$, then these sequences are said to be strongly cofinal, written $E \approx F$.

Take a $\mu$-unital sequence $E=\left\{E_{n}\right\}_{n}$ and form the restricted product measure $\hat{\nu}_{E}$. As the infinite product of $\left\{\mu\left(E_{n}\right)\right\}_{n}$ absolutely converges, $\nu_{E}:=\prod_{n=1}^{\infty} \mu\left(E_{n}\right) \hat{\nu}_{E}$ makes sense as a measure on $M^{\infty}$. Moreover, it depends only on the cofinality class of $E$.

It is easy but important to observe that $\nu_{E}\left(M_{E}^{c}\right)=0$, where $M_{E}:=$ $\bigcup_{n=1}^{\infty}\left(M^{n} \times \prod_{k=n+1}^{\infty} E_{k}\right)$, and that the action of $\sigma \in \mathfrak{S}_{\infty}$ on $M^{\infty}, r(\sigma): x \mapsto x \sigma$ leaves $M_{E}$ invariant. It follows that we get the following theorem, which is basic for later discussions.

Theorem 2.1 (cf. [18]). Given a disjoint $\mu$-unital sequence E, there exists a Borel set $D_{E}$ such that

$$
\forall \sigma \neq \mathrm{id}, \quad D_{E} \sigma \cap D_{E}=\emptyset \quad \text { and } \quad M^{\infty}=\sum_{\sigma \in \mathfrak{S}_{\infty}} D_{E} \sigma \bmod \nu_{E} .
$$

Sketch of proof. Take a sequence $\left\{F^{n}\right\}_{n}$ of disjoint $\mu$-unital sequences that are all cofinal to $E$ such that $M_{E} \subseteq \bigcup_{n=1}^{\infty} F^{n} \bmod \nu_{E}$. Now put $D_{E}:=\sum_{n=1}^{\infty} D^{n}$, where $D^{1}:=F^{1} \cap M_{E}, D^{n}:=\left(F^{n} \cap M_{E}\right) \backslash \bigcup_{\sigma \in \mathfrak{S}_{\infty}, k=1}^{n-1}\left(F^{k} \cap M_{E}\right)(n>1)$.

\section{$\S 2.2$. Action of $\operatorname{Diff}_{0}(M)$ from the left and of $\mathfrak{S}_{\infty}$ from the right}

Let $g \in \operatorname{Diff}_{0}(M)$ and $\sigma \in \mathfrak{S}_{\infty}$ act on $M^{\infty}$ in the following manner:

$$
g\left(x_{1}, x_{2}, \ldots\right)=\left(g x_{1}, g x_{2}, \ldots\right), \quad\left(x_{1}, x_{2}, \ldots\right) \sigma=\left(x_{\sigma(1)}, x_{\sigma(2)}, \ldots\right) .
$$

Clearly the actions of $g$ and of $\sigma$ are mutually commutative, and they lead to transformations of $\nu_{E}$, which will be denoted by $g \nu_{E}$ and $\sigma \nu_{E}$ respectively.

Theorem 2.2 (cf. [4]). Given a disjoint $\mu$-unital sequence $E=\left\{E_{n}\right\}_{n}$,

(1) $\nu_{E}$ is $\mathfrak{S}_{\infty}$-invariant, and

(2) $\nu_{E}$ is $\operatorname{Diff}_{0}(M)$-quasi-invariant.

More precisely, the Radon-Nikodym derivative has the form

$$
\frac{d g \nu_{E}}{d \nu_{E}}(x)=\prod_{n=1}^{\infty} \frac{d g \mu}{d \mu}\left(x_{n}\right),
$$

where the infinite product converges in the $\mathrm{L}_{\nu_{E}}^{1}$-sense on each set $B \times \prod_{k=n_{0}+1}^{\infty} E_{k}$ $\left(B \in \mathfrak{B}\left(M^{n_{0}}\right),(\mu \times \cdots \times \mu)(B)<\infty\right.$, and $n_{0}$ is arbitrary). (The above convergence is, of course, equivalent to $\mathrm{L}_{\nu_{E}}^{2}$-convergence for the square roots of the corresponding functions.) 
By the above theorem, we have two unitary representations, $T(g), g \in$ $\operatorname{Diff}_{0}(M)$, and $R(\sigma), \sigma \in \mathfrak{S}_{\infty}$, on $\mathrm{L}_{\nu_{E}}^{2}\left(M^{\infty}\right)$, such that

$$
T(g): f(x) \mapsto \sqrt{\frac{d g \nu_{E}}{d \nu_{E}}}(x) f\left(g^{-1} x\right), \quad R(\sigma): f(x) \mapsto f(x \sigma) .
$$

The representations $T$ and $R$ commute; moreover, they form a dual pair, as will be proved in the next section.

\section{§2.3. Representation space $\mathcal{H}(\Sigma)$}

Using the same notation for $\mu$ and $E$ as before, and taking an irreducible unitary representation $(\Pi, H)$ of $\mathfrak{S}_{\infty}$, where $H$ is the separable representation Hilbert space, we put $\Sigma:=(\mu, E, \Pi)$. Next, take a Borel measurable $H$-valued function $f$ on $M^{\infty}$ having the following property:

$$
f(x \sigma)=\Pi(\sigma)^{-1} f(x) \quad \text { for } \sigma \in \mathfrak{S}_{\infty} .
$$

Put

$$
\|f\|^{2}:=\int_{D_{E}}\|f(x)\|_{H}^{2} \nu_{E}(d x)
$$

Then the space $\mathcal{H}(\Sigma)$ of those functions $f$ such that $\|f\|<\infty$ forms a Hilbert space with the above norm.

It is useful to note that, for each $f \in \mathcal{H}(\Sigma)$, the integration set $D_{E}$ may be replaced by an arbitrary Borel set $D$ with the following two properties:

(1) $D \sigma \cap D=\emptyset$ if $\sigma\left(\in \mathfrak{S}_{\infty}\right) \neq$ id.

(2) $f=0$ on $\left(\sum_{\sigma \in \mathfrak{S}_{\infty}} D \sigma\right)^{c} \bmod \nu_{E}$.

It follows that the following action $T$ (denoted by the same letter, since no confusion can arrise) of $\operatorname{Diff}_{0}(M)$ on $\mathcal{H}(\Sigma)$ is well-defined and $(T, \mathcal{H}(\Sigma))$ is a unitary representation:

$$
T(g): f(x) \mapsto \sqrt{\frac{d g \nu_{E}}{d \nu_{E}}}(x) f\left(g^{-1} x\right) .
$$

We introduce a technical condition (cc) in the next lemma, which asserts the irreducibility of the natural representation $(T, \mathcal{H}(\Sigma))$.

Lemma 2.1 (cf. [18]). Assume that $d:=\operatorname{dim}(M) \geq 3$ and let $M$ satisfy the following condition:

(cc) There exists a sequence $\left\{U_{n}\right\}_{n}$ of relatively compact, open sets $U_{n} \uparrow M$ such that $\left(\overline{U_{n}}\right)^{c}$ is connected for every $n$. 
Given a disjoint unital sequence $E=\left\{E_{n}\right\}_{n}$, there exists a disjoint $\mu$-unital sequence $G=\left\{G_{n}\right\}_{n}$ that is cofinal to $E$ and has the following properties:

(1) for all $n, G_{n}$ is a relatively compact, open, connected set and $\mu\left(\overline{G_{n}} \backslash G_{n}\right)=0$,

(2) for all $n,\left(\overline{\sum_{k=n+1}^{\infty} G_{k}}\right)^{c}$ is connected,

(3) $\bar{G}_{n} \cap \bar{G}_{m}=\emptyset$ if $n \neq m$,

(4) given a compact set $K$, there exists $N_{K} \in \mathbb{N}$ such that $K \cap G_{n}=\emptyset$ for all $n \geq N_{K}$.

Moreover given any $\epsilon>0$, we may take $G$ such that $\sum_{n=1}^{\infty} \mu\left(E_{n} \ominus G_{n}\right)<\epsilon$.

Proof. We will proceed in several steps.

Step 1. Let $\alpha:=\inf _{n} \mu\left(E_{n}\right)$. For each $E_{n}$, we take a compact set $K_{n}^{(1)}$ satisfying

$$
K_{n}^{(1)} \subseteq E_{n} \quad \text { and } \quad \mu\left(E_{n} \backslash K_{n}^{(1)}\right)<\epsilon_{n},
$$

where $\left\{\epsilon_{n}\right\}_{n}$ is a positive sequence such that $\epsilon_{n}<\alpha / 4$ and $\sum_{n=1}^{\infty} \epsilon_{n}<\epsilon / 4$. It is obvious that

$K^{(1)}:=\left\{K_{n}^{(1)}\right\}_{n} \sim E$ is a disjoint $\mu$-unital sequence, and $\mu\left(K_{n}^{(1)}\right)>\alpha / 4$ for all $n$.

Step 2. Take a sequence $\left\{U_{n}\right\}_{n}$ as in condition (cc). Further, take an increasing sequence $\left\{k_{n}\right\}_{n}$ with

$$
\sum_{i=k_{n}}^{\infty} \mu\left(K_{i} \cap \bar{U}_{n}\right)<\epsilon_{n}
$$

Next for $k_{n} \leq i<k_{n+1}$ we take a compact subset $K_{i}^{(2)}$ in $K_{i}^{(1)} \cap\left(\bar{U}_{n}\right)^{c}$ such that

$$
\mu\left(K_{i}^{(1)} \cap\left(\bar{U}_{n}\right)^{c} \backslash K_{i}^{(2)}\right)<\min \left(\frac{\alpha}{4}-\epsilon_{n}, \frac{\epsilon_{n}}{k_{n+1}-k_{n}}\right),
$$

and for $i \leq k_{1}-1$ we set $K_{i}^{(2)}:=K_{i}^{(1)}$. It is easy to see that $\mu\left(K_{i}^{(2)}\right)>\alpha / 4$ and

$$
\sum_{i=1}^{\infty} \mu\left(K_{i}^{(1)} \backslash K_{i}^{(2)}\right)=\sum_{n=1}^{\infty} \sum_{i=k_{n}}^{k_{n+1}-1} \mu\left(K_{i}^{(1)} \backslash K_{i}^{(2)}\right)<2 \sum_{n=1}^{\infty} \epsilon_{n}<\frac{\epsilon}{2} .
$$

In addition, for a given compact set $L$, we have $K_{n}^{(2)} \cap L=\emptyset$ for all $n \geq k_{n_{0}}$, where $n_{0}$ is such that $L \subset U_{n_{0}}$. Therefore, $\sum_{i \in I} K_{i}^{(2)}$ is closed for any index set $I \subseteq \mathbb{N}$.

Step 3. Take open sets $O_{1}$ and $O_{2}^{\prime}$ that satisfy

$$
O_{1} \cap O_{2}^{\prime}=\emptyset, \quad K_{1}^{(2)} \subset O_{1}, \quad \text { and } \quad \sum_{i=2}^{\infty} K_{i}^{(2)} \subset O_{2}^{\prime} .
$$


Of course we may assume that $\bar{O}_{1}$ is compact. By induction, suppose that we have already chosen relatively compact, open sets $O_{i}(i=1, \ldots, n-1)$ that satisfy

$$
K_{i}^{(2)} \subset O_{i}, \quad \bar{O}_{i} \cap \bar{O}_{j}=\emptyset \quad \text { if } i \neq j, \quad\left(\bigcup_{i=1}^{n-1} \bar{O}_{i}\right) \cap\left(\bigcup_{i=n}^{\infty} K_{i}^{(2)}\right)=\emptyset .
$$

Then there exist open sets $O_{n}$ and $O_{n+1}^{\prime}$ that satisfy

$$
K_{n}^{(2)} \subset O_{n}, \quad\left(\bigcup_{i=1}^{n-1} \bar{O}_{i}\right) \cup\left(\bigcup_{i=n+1}^{\infty} K_{i}^{(2)}\right) \subset O_{n+1}^{\prime}, \quad O_{n} \cap O_{n+1}^{\prime}=\emptyset .
$$

Now the induction may proceed to the next stage. Moreover since $K_{i}^{(2)} \subset\left(\bar{U}_{n}\right)^{c}$ for all $k_{n} \leq i<k_{n+1}$, we may assume that

$$
K_{i}^{(2)} \subset O_{i} \subset \bar{O}_{i} \subset\left(\bar{U}_{n}\right)^{c} \text { and } \sum_{i=1}^{\infty} \mu\left(O_{i} \backslash K_{i}^{(2)}\right)<\frac{\epsilon}{8},
$$

and finally, that each $O_{i}$ is a finite union of open sets that are diffeomorphic to disks in $\mathbb{R}^{d}$. In addition, we use regularity of $\mu$ and Riemann's method of quadrature. Then within small $\mu$-mass gaps, we can deform $O_{i}$ to a finite disjoint union of connected, open sets $O_{i, j}$, where $O_{i, j}$ is included in a neighbourhood diffeomorphic to $\mathbb{R}^{d}$ and it is a union of sets diffeomorphic to cubes in $\mathbb{R}^{d}$. Thus, we have checked all the properties listed in this lemma, except for connectedness.

Step 4. We connect the components $O_{i, j}$ by some polygonal lines included in $\left(\bar{U}_{n}\right)^{c}$. Next, we enlarge these curves to slim open tubes and get a connected open tubular neighbourhood $G_{i}$ by adding those to $O_{i, j}$ or removing them. By property (4) which has already been shown, we can achieve that $\bar{G}_{n}(n=1,2, \ldots)$ are mutually disjoint and that $\left(\sum_{k=n+1}^{\infty} \bar{G}_{n}\right)^{c}$ is connected for each $n$.

When we connect $O_{i, j}$ by slim open tubes, it might happen that the tubes contact to $G_{k}(k<i)$ that have already been constructed. However, if $d \geq 3$, we can go through $G_{k}$ by the slim tubes or by slimmer ones without loss of connectedness. This is the reason why we require the condition $d \geq 3$.

Of course, we can take $\left\{G_{n}\right\}_{n}$ such that $\sum_{n=1}^{\infty} \mu\left(G_{n} \ominus O_{n}\right)<\epsilon / 8$. Hence,

$$
\begin{aligned}
\sum_{n=1}^{\infty} \mu\left(E_{n} \ominus G_{n}\right)< & \sum_{n=1}^{\infty} \mu\left(E_{n} \ominus K_{n}^{(1)}\right)+\sum_{n=1}^{\infty} \mu\left(K_{n}^{(1)} \ominus K_{n}^{(2)}\right) \\
& +\sum_{n=1}^{\infty} \mu\left(K_{n}^{(2)} \ominus O_{n}\right)+\sum_{n=1}^{\infty} \mu\left(O_{n} \ominus G_{n}\right) \\
& <\frac{\epsilon}{4}+\frac{\epsilon}{2}+\frac{\epsilon}{8}+\frac{\epsilon}{8}=\epsilon .
\end{aligned}
$$


Counter-example to (cc). Let $B$ be an open set in $\mathbb{R}^{d}(d \geq 3)$ surrounded by an outer large sphere and including an inner small sphere. The manifold $B$ does not satisfy (cc).

Note that $M=\bigcup U_{n}$ is required in condition (cc), but it is actually sufficient to assume the following weaker (mcc) to obtain irreducibility:

(mcc) There exists a closed set $S$ in $M$ such that $\mu(S)=0, M \backslash S$ is connected and satisfies condition (cc).

Thus, the following theorem concludes this section (cf. [4]).

Theorem 2.3. Let $E$ be a disjoint $\mu$-unital sequence, and $(\Pi, H)$ an irreducible unitary representation of $\mathfrak{S}_{\infty}$. Form a triplet $\Sigma=(E, \mu, \Pi)$ as before. If $\operatorname{dim}(M)$ $\geq 3$ and $M$ satisfies condition (mcc), then the unitary representation $(T, \mathcal{H}(\Sigma))$ is irreducible.

As mentioned in the Introduction, another proof of the above theorem will be given in the Appendix (§A.1).

\section{$\S 3$. Factor representations and dual pairs}

\section{$\S 3.1$. Factor representation}

Let us begin with the following lemma.

Lemma 3.1. Let $M$ be a smooth manifold with $\operatorname{dim}(M) \geq 2$, and let $\mu$ be a locally Euclidean smooth $\sigma$-finite measure on $M$. Take the product measure $\mu^{n}$ on $M^{n}(n \in \mathbb{N})$, and define the natural representation $T$ of $\operatorname{Diff}_{0}(M)$ and a representation $R$ of $\mathfrak{S}_{n}$ on $\mathrm{L}_{\mu^{n}}^{2}\left(M^{n}\right)$ by

$$
\begin{aligned}
& T(g): f\left(x_{1}, \ldots, x_{n}\right) \mapsto \prod_{k=1}^{n} \sqrt{\frac{d g \mu}{d \mu}}\left(x_{k}\right) f\left(g^{-1} x_{1}, \ldots, g^{-1} x_{n}\right), \\
& R(\sigma): f\left(x_{1}, \ldots, x_{n}\right) \mapsto f\left(x_{\sigma(1)}, \ldots, x_{\sigma(n)}\right) .
\end{aligned}
$$

These then form a dual pair. That is, the von Neumann algebra $\left(R(\sigma), \sigma \in \mathfrak{S}_{n}\right)^{\prime \prime}$ generated by $R(\sigma), \sigma \in \mathfrak{S}_{n}$, is the commutant $\left(T(g), g \in \operatorname{Diff}_{0}(M)\right)^{\prime}$ of the von Neumann algebra generated by $T(g), g \in \operatorname{Diff}_{0}(M)$.

Proof. Take disjoint, open, connected sets $U_{1}, \ldots, U_{n}$ in $M$ such that $\mu\left(\overline{U_{k}} \backslash U_{k}\right)$ $=0(k=1, \ldots, n)$, and put $U_{\infty}:=M \backslash \bigcup_{k=1}^{n} U_{k}$. Then

$$
\mathrm{L}_{\mu^{n}}^{2}\left(M^{n}\right)=\sum^{\oplus} \mathrm{L}_{\mu}^{2}\left(U_{q_{1}}\right) \otimes \cdots \otimes \mathrm{L}_{\mu}^{2}\left(U_{q_{n}}\right),
$$


where $q_{k}=1, \ldots, n$ or $\infty$. Let $P_{q}$ be the orthogonal projection from $\mathrm{L}_{\mu^{n}}^{2}\left(M^{n}\right)$ to $\mathrm{L}_{\mu}^{2}\left(U_{q_{1}}\right) \otimes \cdots \otimes \mathrm{L}_{\mu}^{2}\left(U_{q_{n}}\right)$.

Given $A \in\left(T(g), g \in \operatorname{Diff}_{0}(M)\right)^{\prime}$, we put

$$
A_{U}:=A \mid \mathrm{L}_{\mu}^{2}\left(U_{1}\right) \otimes \cdots \otimes \mathrm{L}_{\mu}^{2}\left(U_{n}\right) .
$$

As the natural representation on $\bigotimes_{k=1}^{n} \mathrm{~L}_{\mu}^{2}\left(U_{k}\right)$ of the group $\prod_{k=1}^{n} \operatorname{Diff}_{0}\left(U_{k}\right)$ is irreducible, and as $P_{q} A_{U}$ is an intertwining operator between the corresponding spaces, it follows that $P_{q} A_{U}=0$ except for $q_{k}=\sigma(k)(k=1, \ldots, n)$ for some $\sigma \in \mathfrak{S}_{n}$. Let us denote $P_{q}$ by $P_{\sigma}$ in this case only. Then

$$
P_{\sigma} A_{U}=a_{\sigma, U} R(\sigma) \quad \text { for some } a_{\sigma, U} \in \mathbb{C}
$$

for each $\sigma \in \mathfrak{S}_{n}$, and it follows that

$$
A_{U}=\sum_{\sigma \in \mathfrak{S}_{n}} a_{\sigma, U} R(\sigma) \quad \text { on } \bigotimes_{k=1}^{n} \mathrm{~L}_{\mu}^{2}\left(U_{k}\right)
$$

If $\left\{U_{k}\right\}_{k}$ and $\left\{V_{k}\right\}_{k}$ are two sequences of open sets as above and $U_{k} \cap V_{k} \neq \emptyset$ for all $k$, then we have $a_{\sigma, U}=a_{\sigma, V}$ for all $\sigma \in \mathfrak{S}_{n}$. Finally, the connectedness assumption on $M$ with $\operatorname{dim}(M) \geq 2$ leads to the independence of $a_{\sigma, U}$ from $U$, because we can connect any two points in $\tilde{M}^{n}:=\left\{\left(x_{1}, \ldots, x_{n}\right) \mid x_{i} \neq x_{j}\right.$ for all $\left.i \neq j\right\}$ with a continuous curve in $\tilde{M}^{n}$, and it follows that $a_{\sigma}:=a_{\sigma, U}$. As $\bigotimes_{k=1}^{n} \mathrm{~L}_{\mu}^{2}\left(U_{k}\right)$ generates the whole space, letting $\left\{U_{1}, \ldots, U_{n}\right\}$ run through sequences of disjoint connected open sets, due to the regularity of $\mu$ and $\mu^{n}\left(M^{n} \backslash \tilde{M}^{n}\right)=0$ (cf. [20]) we get

$$
A=\sum_{\sigma \in \mathfrak{S}_{n}} a_{\sigma} R(\sigma)
$$

and $\left(T(g), g \in \operatorname{Diff}_{0}(M)\right)^{\prime} \subseteq\left(R(\sigma), \sigma \in \mathfrak{S}_{n}\right)^{\prime \prime}$. The reverse inclusion is obvious.

Theorem 3.1. Take the Hilbert space $\mathrm{L}_{\nu_{E}}^{2}\left(M^{\infty}\right)$ considered in Section 2. In addition, let $M$ satisfy condition $(\mathrm{mcc})$ and let $\operatorname{dim}(M) \geq 3$. Then the representation $\left(T(g) R(\sigma), \mathrm{L}_{\nu_{E}}^{2}\left(M^{\infty}\right)\right), g \in \operatorname{Diff}_{0}(M), \sigma \in \mathfrak{S}_{\infty}$, is irreducible.

Proof. Take a closed set $S$ as in (mcc) and put $M^{\prime}:=M \cap S^{c}$. The unitary operator $I$ defined by

$$
I: f(x) \in \mathrm{L}_{\nu_{E}}^{2}\left(M^{\prime \infty}\right) \mapsto \prod_{k=1}^{\infty} \chi_{M^{\prime}}\left(x_{k}\right) f(x) \in \mathrm{L}_{\nu_{E}}^{2}\left(M^{\infty}\right)
$$

will be used to reduce the proof to the case of $M^{\prime}$, where $\chi_{M^{\prime}}$ denotes the indicator function of $M^{\prime}$. Thus, in what follows, we assume that condition (cc) on $M$ holds, 
and apply Lemma 2.1. In addition, we replace the letter $G$ in that lemma by $E$ and set

$$
E_{\infty, n}:=\left(\overline{\sum_{k=n+1}^{\infty} E_{k}}\right)^{c}, \quad \hat{E}^{n}:=E_{\infty, n} \times \cdots \times E_{\infty, n} \times E_{n+1} \times E_{n+2} \times \cdots
$$

$\left(E_{\infty, n}\right.$ appears $n$ times in the above expression, and it is a connected, open set in $M$ by condition (2) in Lemma 2.1), and

$$
P_{n}: f \in \mathrm{L}_{\nu_{E}}^{2}\left(M^{\infty}\right) \mapsto \chi_{\hat{E}^{n}} \cdot f \in \mathrm{L}_{\nu_{E}}^{2}\left(M^{\infty}\right) .
$$

Then $P_{n}$ is an increasing projection for each $n$ and tends strongly to Id.

Given $A \in\left(T(g), g \in \operatorname{Diff}_{0}(M)\right)^{\prime}$ and $n \in \mathbb{N}$, define an operator $A_{n} \equiv A_{n}(u, v)$ on $\mathrm{L}_{\mu^{n}}^{2}\left(E_{\infty, n}^{n}\right)$ for each $u, v \in \mathrm{L}_{\mu \times \mu \times \cdots}^{2}\left(\prod_{k=n+1}^{\infty} E_{k}\right)$ as

$$
A_{n}(f):=\int_{\prod_{k=n+1}^{\infty} E_{k}} A(f \otimes u)\left(x_{1}, x_{2}, \ldots\right) \overline{v\left(x_{n+1}, x_{n+2}, \ldots\right)} \prod_{k=n+1}^{\infty} \mu\left(d x_{k}\right) .
$$

$A_{n}$ is characterized as a bounded operator on $\mathrm{L}_{\mu^{n}}^{2}\left(E_{\infty, n}^{n}\right)$ such that

$$
\left\langle A_{n}(f), h\right\rangle_{\mathrm{L}_{\mu}^{2}\left(E_{\infty, n}^{n}\right)}=\langle A(f \otimes u), h \otimes v\rangle_{\mathrm{L}_{\nu_{E}}^{2}\left(M^{\infty}\right)}
$$

for all $h \in \mathrm{L}_{\mu^{n}}^{2}\left(E_{\infty, n}^{n}\right)$. It follows that $T(g) A_{n}=A_{n} T(g)$ for all $g \in \operatorname{Diff}_{0}\left(E_{\infty, n}\right)$, and in view of Lemma 3.1, there exist constants $\lambda_{n}(\sigma, u, v) \in \mathbb{C}$ such that

$$
A_{n}(u, v)=\sum_{\sigma \in \mathfrak{S}_{n}} \lambda_{n}(\sigma, u, v) R(\sigma) \quad \text { on } \mathrm{L}_{\mu^{n}}^{2}\left(E_{\infty, n}^{n}\right) .
$$

As $R(\sigma), \sigma \in \mathfrak{S}_{n}$, are linearly independent, and as $A_{n}(u, v)$ is bounded for all $u, v$, we obtain a bounded operator $B_{n}^{\sigma}$ on $\mathrm{L}_{\mu \times \mu \times \cdots}^{2}\left(\prod_{k=n+1}^{\infty} E_{k}\right)$ that satisfies

$$
\left\langle B_{n}^{\sigma} u, v\right\rangle_{\mathrm{L}_{\mu \times \mu \times \ldots}^{2}\left(\prod_{k=n+1}^{\infty} E_{k}\right)}=\lambda_{n}(\sigma, u, v) \quad \text { for all } \sigma \in \mathfrak{S}_{n} .
$$

Therefore,

$$
\langle A(f \otimes u), h \otimes v\rangle_{\mathrm{L}_{\nu_{E}(M \infty)}^{2}}=\sum_{\sigma \in \mathfrak{S}_{n}}\langle R(\sigma) f, h\rangle_{\mathrm{L}_{\mu}^{2}\left(E_{\infty, n}^{n}\right)}\left\langle B_{n}^{\sigma} u, v\right\rangle_{\mathrm{L}_{\mu \times \mu \times \ldots}^{2} \ldots\left(\prod_{k=n+1}^{\infty} E_{k}\right)} .
$$

In other words,

$$
P_{n} A=\sum_{\sigma \in \mathfrak{S}_{n}} R(\sigma) \otimes B_{n}^{\sigma} \quad \text { on } P_{n}\left(\mathrm{~L}_{\nu_{E}}^{2}\left(M^{\infty}\right)\right) .
$$

Now take another diffeomorphism $g$ from the restricted product group $\prod_{k=n+1}^{\infty *} \operatorname{Diff}_{0}\left(E_{k}\right)$. It then follows from the trivial relation $P_{n} T(g)=T(g) P_{n}$ that

$$
T(g) B_{n}^{\sigma}=B_{n}^{\sigma} T(g) \quad \text { for all } \sigma \in \mathfrak{S}_{n} .
$$


As has already been noted, by the irreducibility of the natural representation of $\prod_{k=n+1}^{\infty *} \operatorname{Diff}_{0}\left(E_{k}\right)$ on $\mathrm{L}_{\mu \times \mu \times \cdots}^{2}\left(\prod_{k=n+1}^{\infty} E_{k}\right)$ (cf. [18]), $B_{n}^{\sigma}$ is a scalar operator $\lambda_{n, \sigma}$ Id for each $\sigma \in \mathfrak{S}_{n}$. Consequently,

$$
P_{n} A P_{n}=\left(\sum_{\sigma \in \mathfrak{S}_{n}} \lambda_{n, \sigma} R(\sigma)\right) P_{n},
$$

where the symbol $R(\sigma)$ is used for both $\mathrm{L}_{\mu^{n}}^{2}\left(E_{\infty, n}^{n}\right)$ and $\mathrm{L}_{\nu_{E}}^{2}\left(M^{\infty}\right)$, since no confusion can arise. Fix $N \in \mathbb{N}$ for a while. Further, take $n_{0} \geq N$ and a Borel set $B_{0}$ of product type such that

$$
\begin{aligned}
& B_{0}:=U_{1} \times \cdots \times U_{n_{0}} \times E_{n_{0}+1} \times E_{n_{0}+2} \times \cdots, \\
& B_{0} \subset \hat{E}^{N}, \quad U_{i} \cap U_{j}=\emptyset \quad\left(\forall i \neq j \leq n_{0}\right), \\
& \forall i, \quad 0<\mu\left(U_{i}\right)<\infty .
\end{aligned}
$$

Consequently, $U_{i} \cap E_{k}=\emptyset$ for $1 \leq i \leq n_{0}$ and $k \geq n_{0}+1$, and it follows that $B_{0} \sigma \cap B_{0}=\emptyset$ for $\sigma(\neq \mathrm{id}) \in \mathfrak{S}_{\infty}$, and $\chi_{B_{0}} \in P_{n}\left(\mathrm{~L}_{\nu_{E}}^{2}\left(M^{\infty}\right)\right)$ for all $n \geq n_{0}$. Now applying (3.1) to $\chi_{B_{0}}$, we obtain

$$
\left\|P_{n} A P_{n} \chi_{B_{0}}\right\|^{2}=\sum_{\sigma \in \mathfrak{S}_{n}}\left|\lambda_{n, \sigma}\right|^{2} \nu_{E}\left(B_{0}\right),
$$

and this implies

$$
\sum_{\sigma \in \mathfrak{S}_{n}}\left|\lambda_{n, \sigma}\right|^{2} \leq\|A\|^{2}
$$

Next, from the assumption $\left.A \in\left(R(\sigma), \sigma \in \mathfrak{S}_{\infty}\right)\right)^{\prime}$ and from the fact that $P_{n} R(\sigma)=R(\sigma) P_{n}$ for $\sigma \in \mathfrak{S}_{n}$, it follows that

$$
\lambda_{n, \sigma}=\lambda_{n, \tau \sigma \tau^{-1}} \quad \text { for all } \sigma, \tau \in \mathfrak{S}_{n} .
$$

Take any $k \in \mathbb{N}$ and $\sigma_{0} \in \mathfrak{S}_{k}$ not equal to id. As can be easily seen, for any $l \in \mathbb{N}$ greater than $k$, there exist $\tau_{1}, \ldots, \tau_{m} \in \mathfrak{S}_{l}$ such that $\tau_{i}^{-1} \sigma_{0} \tau_{i}$ are mutually distinct $(i=1, \ldots, m)$, where $m$ is the greatest integer smaller than $l / k$. Thus,

$$
\left|\lambda_{l, \sigma_{0}}\right|^{2} \leq\|A\|^{2} / m \rightarrow 0 \quad(l \rightarrow \infty) .
$$

Hence, for any $\sigma \neq \mathrm{id} \in \mathfrak{S}_{\infty}$,

$$
\left\langle A \chi_{B_{0}}, \chi_{B_{0} \sigma}\right\rangle=\lim _{l \rightarrow \infty}\left\langle P_{l} A \chi_{B_{0}}, \chi_{B_{0} \sigma}\right\rangle=\lim _{l \rightarrow \infty} \lambda_{l, \sigma^{-1}} \nu_{E}\left(B_{0}\right)=0,
$$

while

$$
\left\langle A \chi_{B_{0}}, \chi_{B_{0}}\right\rangle=\lim _{l \rightarrow \infty}\left\langle P_{l} A \chi_{B_{0}}, \chi_{B_{0}}\right\rangle=\lim _{l \rightarrow \infty} \lambda_{l, \mathrm{id}} \nu_{E}\left(B_{0}\right) .
$$

Thus, we have a limit of $\left\{\lambda_{l, \text { id }}\right\}_{l}$ which is denoted by $\lambda$. 
Note that the indicator functions of the Borel sets as in (3.2) generate the space $\mathrm{L}_{\nu_{E}}^{2}\left(\hat{E}^{N}\right)$. Take finitely many such Borel sets $B_{1}, \ldots, B_{s}$ of $\hat{E}^{n}$ and complex constants $c_{1}, \ldots, c_{s}$. Then

$$
\begin{aligned}
\left\langle A\left(\sum_{i=1}^{s} c_{i} \chi_{B_{i}}\right), \sum_{i=1}^{s} c_{i} \chi_{B_{i}}\right\rangle & =\lim _{l \rightarrow \infty}\left\langle P_{l} A\left(\sum_{i=1}^{s} c_{i} \chi_{B_{i}}\right), \sum_{i=1}^{s} c_{i} \chi_{B_{i}}\right\rangle \\
& =\lim _{l \rightarrow \infty} \sum_{\sigma \in \mathfrak{S}_{l}} \sum_{i, j}^{s} \lambda_{l, \sigma} c_{i} \overline{c_{j}}\left\langle\chi_{B_{i} \sigma^{-1}}, \chi_{B_{j}}\right\rangle .
\end{aligned}
$$

Since $\left\langle\chi_{B_{i} \sigma^{-1}}, \chi_{B_{j}}\right\rangle=0$ except for $\sigma \in \mathfrak{S}_{N}$,

$$
\begin{aligned}
\left\langle A\left(\sum_{i=1}^{s} c_{i} \chi_{B_{i}}\right), \sum_{i=1}^{s} c_{i} \chi_{B_{i}}\right\rangle & =\sum_{\sigma \in \mathfrak{S}_{N}} \lim _{l \rightarrow \infty} \sum_{i, j}^{s} \lambda_{l, \sigma} c_{i} \overline{c_{j}}\left\langle\chi_{B_{i} \sigma^{-1}}, \chi_{B_{j}}\right\rangle \\
& =\lambda \sum_{i, j}^{s} c_{i} \overline{c_{j}}\left\langle\chi_{B_{i}}, \chi_{B_{j}}\right\rangle .
\end{aligned}
$$

Hence, $\langle A f, f\rangle=\lambda\langle f, f\rangle$ for any $f=\sum_{i=1}^{s} c_{i} \chi_{B_{i}}$. As $N$ is arbitrary, this shows that $A$ is a scalar operator.

Corollary 3.1. Both $\left(T(g), \mathrm{L}_{\nu_{E}}^{2}\left(M^{\infty}\right)\right), g \in \operatorname{Diff}_{0}(M)$, and $\left(R(\sigma), \mathrm{L}_{\nu_{E}}^{2}\left(M^{\infty}\right)\right)$, $\sigma \in \mathfrak{S}_{\infty}$, are factor representations.

Proof. In fact, $\left(T(g), \operatorname{Diff}_{0}(M)\right)^{\prime \prime} \subseteq\left(R(\sigma), \sigma \in \mathfrak{S}_{\infty}\right)^{\prime}$ and $\left(R(\sigma), \sigma \in \mathfrak{S}_{\infty}\right)^{\prime \prime} \subseteq$ $\left(T(g), \operatorname{Diff}_{0}(M)\right)^{\prime}$. Thus, the conclusion follows from the above theorem.

Theorem 3.1 and Corollary 3.1 indicate that $T$ and $R$ behave as in the finitedimensional case (cf. [20]).

Theorem 3.2. Using the same assumptions and notation as in Theorem 3.1, let F be another disjoint $\mu$-unital sequence. The representations $\left(T(g) R(\sigma), \mathrm{L}_{\nu_{E}}^{2}\left(M^{\infty}\right)\right)$ and $\left(T(g) R(\sigma), \mathrm{L}_{\nu_{F}}^{2}\left(M^{\infty}\right)\right)$ are equivalent if and only if there exists a permutation a of $\mathbb{N}$ (maybe infinite) such that $E \sim F a^{-1}$.

Proof. This is an immediate consequence of Theorem B in the Appendix.

Theorem 3.3. $\left(R(\sigma), \mathrm{L}_{\nu_{E}}^{2}\left(M^{\infty}\right)\right), \sigma \in \mathfrak{S}_{\infty}$, and $\left(T(g), \mathrm{L}_{\nu_{E}}^{2}\left(M^{\infty}\right)\right), g \in \operatorname{Diff}_{0}(M)$, are factor representations of type $\mathrm{II}_{1}$ and $\mathrm{II}_{\infty}$, respectively.

Proof. Let $\ell^{2}\left(\mathfrak{S}_{\infty}\right)$ be the representation space of the right regular representation of $\mathfrak{S}_{\infty}$, and denote the indicator function of the set $\{\sigma\}$ consisting of a single element $\sigma \in \mathfrak{S}_{\infty}$ by $e_{\sigma}$. It is well-known that the von Neumann algebra $\mathcal{M}$ generated 
by the right regular representation is of type $\mathrm{II}_{1}$, and that its relative dimensionality function $d_{\mathcal{M}}$ is given by

$$
d_{\mathcal{M}}(M):=\left\langle P_{M} e_{\mathrm{id}}, e_{\mathrm{id}}\right\rangle_{\ell^{2}\left(\mathfrak{S}_{\infty}\right)} \quad \text { for all } P_{M} \in \mathcal{M}
$$

Now, take the set $D_{E}$ introduced in Section 2, take a c.o.n.s. $\left\{h_{n}\right\}_{n}$ in $\mathrm{L}_{\nu_{E}}^{2}\left(D_{E}\right)$, and finally, take the Hilbert space $H_{n}$ spanned by $R(\sigma) h_{n}, \sigma \in \mathfrak{S}_{\infty}$. Then

$$
\mathrm{L}_{\nu_{E}}^{2}\left(M^{\infty}\right)=\sum^{\oplus} H_{n}
$$

and $\ell^{2}\left(\mathfrak{S}_{\infty}\right)$ and $H_{n}$ are isomorphic through the following intertwining unitary operator $\eta_{n}$ :

$$
\eta_{n}: e_{\sigma} \in \ell^{2}\left(\mathfrak{S}_{\infty}\right) \mapsto R(\sigma)^{-1} h_{n} \in H_{n} \quad \text { for all } \sigma \in \mathfrak{S}_{\infty}
$$

Let $Q_{n}$ be the orthogonal projection from $\mathrm{L}_{\nu_{E}}^{2}\left(M^{\infty}\right)$ to $H_{n}$. It is easy to see that

$$
T \in \mathcal{R}:=\left(R(\sigma), \sigma \in \mathfrak{S}_{\infty}\right)^{\prime \prime} \Rightarrow \eta_{n}^{-1} Q_{n} T \eta_{n} \in \mathcal{M} \text { for all } n .
$$

Note that if $T=P_{M}$ is a projection, $Q_{n} P_{M}$ is also a projection in $H_{n}$. As a result, we can define a relative dimensionality function $d_{\mathcal{R}}$ on $\mathcal{R}$ by

$$
d_{\mathcal{R}}(M):=\left\langle Q_{n} P_{M} h_{n}, h_{n}\right\rangle_{H_{n}} \quad \text { for all } P_{M} \in\left(R(\sigma), \sigma \in \mathfrak{S}_{\infty}\right)^{\prime \prime},
$$

which obviously does not depend on $n \in \mathbb{N}$. This demonstrates the first assertion.

Next, consider the factor representation $T$, and use the fact that $T(g), g \in$ $\operatorname{Diff}_{0}(M)$, and $R(\sigma), \sigma \in \mathfrak{S}_{\infty}$, form a dual pair, as claimed in [5]. However, due to a mistake in the uniform estimate of a norm in [5, Lemma 5.5], we will give a corrected proof in the next subsection.

By the result on dual pairs,

$$
Q_{n} \in\left(R(\sigma), \sigma \in \mathfrak{S}_{\infty}\right)^{\prime}=\left(T(g), g \in \operatorname{Diff}_{0}(M)\right)^{\prime \prime}=: \mathcal{T} .
$$

In addition, take a unitary operator $S_{n, m}$ for $n \neq m \in \mathbb{N}$ defined by

$$
S_{n, m}:=\left\{\begin{array}{l}
R(\sigma)^{-1} h_{n} \mapsto R(\sigma)^{-1} h_{m}, \\
R(\sigma)^{-1} h_{m} \mapsto R(\sigma)^{-1} h_{n}, \\
R(\sigma)^{-1} h_{l} \mapsto R(\sigma)^{-1} h_{l} \quad(l \neq n, m) .
\end{array}\right.
$$

It is clear that $S_{n, m} \in \mathcal{T}$ and $S_{n, m}\left(H_{n}\right)=H_{m}$.

As a well-known theorem on coupled factors (cf. [11]) guarantees that $\mathcal{T}$ is of type II, we have only to check that its relative dimensionality function $d_{\mathcal{T}}$ has

$$
d_{\mathcal{T}}\left(\mathrm{L}_{\nu_{E}}^{2}\left(M^{\infty}\right)\right)=\infty
$$

which follows directly from the above arguments. 
§3.2. Dual pairs of $T(g), g \in \operatorname{Diff}_{0}(M)$, and $R(\sigma), \sigma \in \mathfrak{S}_{\infty}$

Theorem 3.4. Under the assumptions of Theorem 3.1, $T(g), g \in \operatorname{Diff}_{0}(M)$, and $R(\sigma), \sigma \in \mathfrak{S}_{\infty}$, form a dual pair.

Proof. Using the same reasonings as in the proof of Theorem 3.1, we can assume that $M$ satisfies condition (cc).

Given $A \in\left(T(g), g \in \operatorname{Diff}_{0}(M)\right)^{\prime}$, relation (3.1) in Theorem 3.1 follows by the same arguments as before. At this stage, the uniform boundedness of the operators $\sum_{\sigma \in \mathfrak{S}_{n}} \lambda_{n, \sigma} R(\sigma)$ on the whole space $\mathrm{L}_{\nu_{E}}^{2}\left(M^{\infty}\right)$ is important. We prove it as follows:

First of all,

$$
\left|\lambda_{n, \sigma}\right| \leq\|A\| \quad \text { for all } n \in \mathbb{N} \text { and } \sigma \in \mathfrak{S}_{n},
$$

by (3.3). In addition, the family $\mathcal{O}:=\left\{\sum_{\sigma \in \mathfrak{S}_{n}} \lambda_{\sigma} R(\sigma)\right\}_{\left|\lambda_{\sigma}\right| \leq\|A\|}$ of operators on $\mathrm{L}_{\mu^{n}}^{2}\left(M^{n}\right)$ is compact in the uniform topology, and given $\epsilon>0$, there exists a compact set $\Omega:=K \times \cdots \times K \subset M^{n}$ such that

$$
\|T\|-\epsilon<\sup _{f \in \mathrm{L}_{\mu^{n}}^{2}\left(M^{n}\right)} \frac{\left\|T\left(f \cdot \chi_{\Omega}\right)\right\|}{\left\|f \cdot \chi_{\Omega}\right\|} \quad \text { for all } T \in \mathcal{O} .
$$

Cover $K$ by open coordinate neighbourhoods $O_{i}^{\prime}(i=1, \ldots, s)$ such that

$$
K \subset O_{1}^{\prime} \cup \cdots \cup O_{s}^{\prime} \quad \text { and } \quad \mu\left(\overline{O_{i}^{\prime}} \backslash O_{i}^{\prime}\right)=0 \quad(i=1, \ldots, s) .
$$

Put

$$
O_{1}:=O_{1}^{\prime}, \quad O_{2}:=O_{2}^{\prime} \backslash \overline{O_{1}^{\prime}}, \ldots, O_{s}:=O_{s}^{\prime} \backslash\left(\overline{O_{1}^{\prime}} \cup \cdots \cup \overline{O_{s-1}^{\prime}}\right)
$$

and

$$
\hat{G}:=O_{1} \cup \cdots \cup O_{s}, \quad \Omega_{1}:=\prod_{k=1}^{n} \hat{G} .
$$

Then $\hat{G}$ is a union of disjoint open sets and $\Omega_{1}$ has the same property (3.4) as $\Omega$.

Finally, reasoning locally on each $O_{i}$, we may find a sequence of disjoint open sets $W_{i}(i=1, \ldots, T)$ diffeomorphic to cubes in $\mathbb{R}^{d}$, and such that their union $G$ approximates $\hat{G}$ from the inside. That is, for any $\delta>0$, we may find such an open set $G$ with $\mu(\hat{G} \backslash G)<\delta$ and $W_{i} \cap W_{j}=\emptyset(i \neq j)$. In addition, after changing $\Omega_{1}$ to $\Omega_{2}:=\prod_{k=1}^{n} G$, we have an equality of type (3.4), if we take $\delta$ sufficiently small:

$$
\|T\|-3 \epsilon<\sup _{f \in \mathrm{L}_{\mu^{n}}^{2}\left(M^{n}\right)} \frac{\left\|T\left(f \cdot \chi_{\Omega_{2}}\right)\right\|}{\left\|f \cdot \chi_{\Omega_{2}}\right\|} \quad \text { for all } T \in \mathcal{O} .
$$

Let us denote the centre of each cube $W_{i}$ in $G$ by $x_{i}(i=1, \ldots, T)$, and take other points $\xi_{1}, \ldots, \xi_{T}$ in $E_{\infty, n}$, mutually distinct. Removing a small mass of $\overline{E_{\infty, n}}$ 
from $\hat{G}$ in advance, as necessary, we may assume $\left\{\xi_{1}, \ldots, \xi_{T}\right\} \cap G=\emptyset$. Second, connect $x_{i}$ and $\xi_{i}$ with open slim tubes $\Gamma_{i}$ that are mutually disjoint, $\Gamma_{i} \cap W_{j}=\emptyset$ $(i \neq j)$ and take maps $\psi_{i} \in \operatorname{Diff}_{0}(M)$ such that supp $\psi_{i} \subset \Gamma_{i}$ and $\psi_{i}\left(x_{i}\right)=\xi_{i}$. Finally, take open sets $V\left(\xi_{i}\right) \subset E_{\infty, n}$, and $U\left(x_{i}\right) \subset W_{i}$ such that $\psi_{i}\left(U\left(x_{i}\right)\right) \subset$ $V\left(\xi_{i}\right)$, and take a map $\phi_{i}$ such that $\phi_{i}\left(W_{i}\right) \subset U\left(x_{i}\right)$ and $\operatorname{supp} \phi_{i} \cap\left(W_{j} \cup \Gamma_{j}\right)=\emptyset$ $(i \neq j)$. Put $\phi:=\prod_{i=1}^{T} \psi_{i} \circ \phi_{i}$. Then $\phi(G) \subset E_{\infty, n}$.

Now return to relation (3.1), and fix $n \in \mathbb{N}$ for a while. Take a function $k_{\lambda} \in \mathrm{L}_{\mu^{n}}^{2}\left(M^{n}\right)$ for each $\lambda:=\left\{\lambda_{n, \sigma}\right\}_{\sigma \in \mathfrak{S}_{n}}$ bounded by $\|A\|$, satisfying

$$
\left\|\sum_{\sigma \in \mathfrak{S}_{n}} \lambda_{n, \sigma} R(\sigma)\right\|-3 \epsilon<\frac{\left\|\left(\sum_{\sigma \in \mathfrak{S}_{n}} \lambda_{n, \sigma} R(\sigma)\right) k_{\lambda} \chi_{\Omega_{2}}\right\|_{\mathrm{L}_{\mu^{n}}^{2}\left(M^{n}\right)}}{\left\|k_{\lambda} \chi_{\Omega_{2}}\right\|_{\mathrm{L}_{\mu}^{2}\left(M^{n}\right)}} .
$$

Next, set

$$
f_{\lambda}(x):=\prod_{k=1}^{n} \sqrt{\frac{d \phi \mu}{d \mu}}\left(x_{k}\right) \frac{\left(k_{\lambda} \cdot \chi_{\Omega_{2}}\right)\left(\phi^{-1} x_{1}, \ldots, \phi^{-1} x_{n}\right)}{\left\|k_{\lambda} \chi_{\Omega_{2}}\right\|_{\mathrm{L}_{\mu^{n}}\left(M^{n}\right)}} \prod_{k=n+1}^{\infty} \chi_{E_{k}}\left(x_{k}\right) .
$$

Then

$$
T(\phi)^{-1} f_{\lambda}(x)=\frac{\left(k_{\lambda} \chi_{\Omega_{2}}\right)\left(x_{1}, \ldots, x_{n}\right)}{\left\|k_{\lambda} \chi_{\Omega_{2}}\right\|_{L_{\mu^{n}}^{2}\left(M^{n}\right)}} \prod_{k=n+1}^{\infty} \sqrt{\frac{d \phi^{-1} \mu}{d \mu}}\left(x_{k}\right) \chi_{E_{k}}\left(\phi\left(x_{k}\right)\right),
$$

and $f_{\lambda} \in P_{n}\left(\mathrm{~L}_{\nu_{E}}^{2}\left(M^{\infty}\right)\right)$. It follows from (3.1) that

$$
\begin{aligned}
T(\phi)^{-1} P_{n} A P_{n} f_{\lambda}(x)= & \left(\sum_{\sigma \in \mathfrak{S}_{n}} \lambda_{n, \sigma} R(\sigma)\right) T(\phi)^{-1} f_{\lambda}(x) \\
= & \left\|k_{\lambda} \chi_{\Omega_{2}}\right\|_{\mathrm{L}_{\mu^{n}}^{2}\left(M^{n}\right)}^{-1}\left(\sum_{\sigma \in \mathfrak{S}_{n}} \lambda_{n, \sigma} R(\sigma)\right)\left(k_{\lambda} \chi_{\Omega_{2}}\right)\left(x_{1}, \ldots, x_{n}\right) \\
& \cdot \prod_{k=n+1}^{\infty} \sqrt{\frac{d \phi^{-1} \mu}{d \mu}}\left(x_{k}\right) \chi_{E_{k}}\left(\phi\left(x_{k}\right)\right) .
\end{aligned}
$$

Hence,

$$
\begin{aligned}
\left\|T(\phi)^{-1} P_{n} A P_{n} f_{\lambda}\right\|_{L_{\nu_{E}}^{2}\left(M^{\infty}\right)}^{2} & >\left(\left\|\sum_{\sigma \in \mathfrak{S}_{n}} \lambda_{n, \sigma} R(\sigma)\right\|-3 \epsilon\right)^{2} \prod_{k=n+1}^{\infty} \mu\left(E_{k}\right) \\
& =\left(\left\|\sum_{\sigma \in \mathfrak{S}_{n}} \lambda_{n, \sigma} R(\sigma)\right\|-3 \epsilon\right)^{2}\left\|f_{\lambda}\right\|_{\mathrm{L}_{\nu_{E}}^{2}\left(M^{\infty}\right)}^{2} .
\end{aligned}
$$

This indicates that

$$
\left\|\sum_{\sigma \in \mathfrak{S}_{n}} \lambda_{n, \sigma} R(\sigma)\right\|<\|A\|+3 \epsilon
$$


In addition, the operator norm of $\sum_{\sigma \in \mathfrak{S}_{n}} \lambda_{n, \sigma} R(\sigma)$ is left invariant under a change of the basic space from $\mathrm{L}_{\mu^{n}}^{2}\left(M^{n}\right)$ to $\mathrm{L}_{\nu_{E}}^{2 n}\left(M^{\infty}\right)$. This gives the uniform boundedness. As a result, we easily see that $\sum_{\sigma \in \mathfrak{S}_{n}} \lambda_{n, \sigma} R(\sigma)$ converges strongly to $A$, and we have $A \in\left(R(\sigma), \sigma \in \mathfrak{S}_{\infty}\right)^{\prime \prime}$. In other words,

$$
\left(T(g), g \in \operatorname{Diff}_{0}(M)\right)^{\prime} \subseteq\left(R(\sigma), \sigma \in \mathfrak{S}_{\infty}\right)^{\prime \prime} .
$$

The reverse inclusion is obvious.

\section{$\S 4$. Irreducible decompositions of $\left(T(g), \mathrm{L}_{\nu_{E}}^{2}\left(M^{\infty}\right)\right), g \in \operatorname{Diff}_{0}(M)$}

As mentioned in the Introduction, an irreducible decomposition of $T$ is provided in this section.

Theorem 4.1. Take the Hilbert space $\mathrm{L}_{\nu_{E}}^{2}\left(M^{\infty}\right)$ considered in Section 2; in addition, let $M$ satisfy condition (mcc) and suppose $\operatorname{dim}(M) \geq 3$. Then the natural representation $\left(T(g), \mathrm{L}_{\nu_{E}}^{2}\left(M^{\infty}\right)\right), g \in \operatorname{Diff}_{0}(M)$, has an irreducible decomposition, and the irreducible components are the spaces $\left(T(g), \mathcal{H}\left(\Sigma_{\lambda}\right)\right)$, where $\mathcal{H}\left(\Sigma_{\lambda}\right)$ are the Hilbert spaces defined in the Introduction, $\Sigma_{\lambda}:=\left(\mu, E, H_{\lambda}\right)$, and $\left(\Pi_{\lambda}, H_{\lambda}\right)$ are irreducible components of the left regular representation of $\mathfrak{S}_{\infty}$.

Proof. The proof is divided into several steps.

Step 1. First, we will give a unitary operator $U_{1}$ from $\mathrm{L}_{\nu_{E}}^{2}\left(M^{\infty}\right)$ onto $\ell^{2}\left(\mathfrak{S}_{\infty}\right) \otimes$ $\mathrm{L}_{\nu_{E}}^{2}\left(D_{E}\right)$.

Fix a c.o.n.s. $\left\{h_{n}(x)\right\}_{n}$ in $\mathrm{L}_{\nu_{E}}^{2}\left(D_{E}\right)$. Of course, $\left\{h_{n}\left(x \sigma^{-1}\right)\right\}_{n}$ forms a c.o.n.s. in $\mathrm{L}_{\nu_{E}}^{2}\left(D_{E} \sigma\right)$ for any $\sigma \in \mathfrak{S}_{\infty}$. Given $n \in \mathbb{N}, f \in \mathrm{L}_{\nu_{E}}^{2}\left(M^{\infty}\right)$ and $\sigma \in \mathfrak{S}_{\infty}$, put

$$
a_{n}(\sigma):=\int_{D_{E} \sigma} f(x) \overline{h_{n}\left(x \sigma^{-1}\right)} \nu_{E}(d x)=\int_{D_{E}} f(x \sigma) \overline{h_{n}(x)} \nu_{E}(d x) .
$$

It follows directly that

$$
\sum_{\sigma \in \mathfrak{S}_{\infty}} \sum_{n=1}^{\infty}\left|a_{n}(\sigma)\right|^{2}<\infty
$$

Define a map $U_{1}$ by

$$
U_{1}(f):=\sum_{n=1}^{\infty} a_{n} \otimes h_{n}
$$

One may easily observe that $U_{1}$ is the desired unitary operator.

Step 2. Let $\left(L(\sigma), \ell^{2}\left(\mathfrak{S}_{\infty}\right)\right), \sigma \in \mathfrak{S}_{\infty}$, be the left regular representation. Decompose it, applying the reduction theory of the von Neumann algebra generated by 
$L(\sigma), \sigma \in \mathfrak{S}_{\infty}$, and by the maximal abelian ring of its commutant (cf. $\left.[8,9,19]\right)$ :

$$
\ell^{2}\left(\mathfrak{S}_{\infty}\right) \sim \int^{\oplus} H_{\lambda} \sqrt{d \sigma(\lambda)}, \quad L(\sigma) \sim \sum \Pi_{\lambda}(\sigma) .
$$

It then follows that there exists a realization of the orthogonal generalized direct sum of the $H_{\lambda} \otimes \mathrm{L}_{\nu_{E}}^{2}\left(D_{E}\right)$ to $\ell^{2}\left(\mathfrak{S}_{\infty}\right) \otimes \mathrm{L}_{\nu_{E}}^{2}\left(D_{E}\right)$ with the weight function $\sigma(\lambda)$ :

$$
\ell^{2}\left(\mathfrak{S}_{\infty}\right) \otimes \mathrm{L}_{\nu_{E}}^{2}\left(D_{E}\right) \sim \int^{\oplus} H_{\lambda} \otimes \mathrm{L}_{\nu_{E}}^{2}\left(D_{E}\right) \sqrt{d \sigma(\lambda)} \sim \int^{\oplus} \mathrm{L}_{\nu_{E}}^{2}\left(D_{E}, H_{\lambda}\right) \sqrt{d \sigma(\lambda)} .
$$

Thus, we have a unitary map

$$
U_{2}: \ell^{2}\left(\mathfrak{S}_{\infty}\right) \otimes \mathrm{L}_{\nu_{E}}^{2}\left(D_{E}\right) \rightarrow \int^{\oplus} \mathrm{L}_{\nu_{E}}^{2}\left(D_{E}, H_{\lambda}\right) \sqrt{d \sigma(\lambda)}
$$

such that

$$
U_{2}\left(\sum_{n} a_{n} \otimes h_{n}\right):=\int_{-} \underline{f}_{\lambda} \sqrt{d \sigma(\lambda)}
$$

where

$$
\underline{f}_{\lambda}(x)=\sum_{n=1}^{\infty} \underline{a_{n, \lambda}} h_{n}(x) \quad \text { for all } x \in D_{E}
$$

we follow the notation of von Neumann [13]. It is evident that $U_{2}$ is actually a unitary operator.

Step 3. Finally, extend the domain $D_{E}$ of each component function $\underline{f}_{\lambda}$ in order to get another function $\underline{F}_{\lambda}$ in $\mathcal{H}\left(\Sigma_{\lambda}\right)$ defined by;

$$
\underline{F}_{\lambda}(x \sigma):=\Pi_{\lambda}^{-1}(\sigma) \underline{f}_{\lambda}(x) \quad \text { for all } x \in D_{E} \text { and } \sigma \in \mathfrak{S}_{\infty} .
$$

In addition, define

$$
\begin{aligned}
& U_{3}:=\int_{-} \underline{f}_{\lambda} \sqrt{d \sigma(\lambda)} \in \int^{\oplus} \mathrm{L}_{\nu_{E}}^{2}\left(D_{E}, H_{\lambda}\right) \sqrt{d \sigma(\lambda)} \\
& \mapsto \int_{-} \underline{F}_{\lambda} \sqrt{d \sigma(\lambda)} \in \int^{\oplus} \mathcal{H}\left(\Sigma_{\lambda}\right) \sqrt{d \sigma(\lambda)} .
\end{aligned}
$$

Step 4. Now let us compose these three maps:

$$
U:=U_{3} \circ U_{2} \circ U_{1} .
$$

It turns out that $U$ is independent of the set $D_{E}$ and of the c.o.n.s. in $\mathrm{L}_{\nu_{E}}^{2}\left(D_{E}\right)$. As the latter is easily checked, we now verify the independence from $D_{E}$.

Take another $D_{F}$ with the same properties as $D_{E}$. For each $\rho \in \mathfrak{S}_{\infty}$, we take a c.o.n.s. $\left\{h_{n}^{\rho}(x)\right\}_{n<N_{\rho}}$ in $\mathrm{L}_{\nu_{E}}^{2}\left(D_{E} \cap D_{F} \rho\right)$, where $N_{\rho}$ is an integer or $\infty$. Of course $\left\{h_{n}^{\rho}(x \rho)\right\}_{n<N_{\rho}}$ is a c.o.n.s. in $\mathrm{L}_{\nu_{E}}^{2}\left(D_{E} \rho^{-1} \cap D_{F}\right)$, and $\left\{h_{n}^{\rho}(x \rho)\right\}_{\rho, n<N_{\rho}}$ is a c.o.n.s. 
in $\mathrm{L}_{\nu_{E}}^{2}\left(D_{F}\right)$. For any $\sigma \in \mathfrak{S}_{\infty}$, set

$$
\begin{aligned}
a_{\rho, n}(\sigma) & :=\int_{D_{E} \cap D_{F} \rho} f(x \sigma) \overline{h_{n}^{\rho}(x)} \nu_{E}(d x), \\
b_{\rho, n}(\sigma) & :=\int_{D_{F} \cap D_{E} \rho^{-1}} f(x \sigma) \overline{h_{n}^{\rho}(x \rho)} \nu_{E}(d x) .
\end{aligned}
$$

Then it follows that

$$
b_{\rho, n}(\sigma)=a_{\rho, n}\left(\rho^{-1} \sigma\right) \quad \text { for all } \sigma, \rho \in \mathfrak{S}_{\infty},
$$

which leads to

$$
\Pi_{\lambda}(\rho) \underline{a_{\rho, n, \lambda}}=\underline{b_{\rho, n, \lambda}} \quad \text { for a.e. } \lambda .
$$

For any $f \in \mathrm{L}_{\nu_{E}}^{2}\left(M^{\infty}\right)$, denote the corresponding element in the cases of $D_{E}$ and $D_{F}$ by

$$
\int_{-} \underline{F}_{\lambda, E} \sqrt{d \sigma(\lambda)} \text { and } \int_{-} \underline{F}_{\lambda, F} \sqrt{d \sigma(\lambda)}
$$

respectively. Then, for any $x \in D_{E}$ and $\sigma \in \mathfrak{S}_{\infty}$,

$$
\underline{F}_{\lambda, E}(x \sigma)=\sum_{\rho, n<N_{\rho}} h_{n}^{\rho}(x) \Pi_{\lambda}^{-1}(\sigma)\left(\underline{a_{\rho, n, \lambda}}\right),
$$

and for any $y \in D_{F}$ and $\tau \in \mathfrak{S}_{\infty}$,

$$
\left.\underline{F}_{\lambda, F}(y \tau)=\sum_{\rho, n<N_{\rho}} h_{n}^{\rho}(y \rho) \Pi_{\lambda}^{-1}(\tau) \underline{\left(b_{\rho, n, \lambda}\right.}\right) .
$$

If $x \sigma=y \tau$, then $x=y \tau \sigma^{-1} \in D_{F} \tau \sigma^{-1}$, and $h_{n}^{\rho}(x)=0$ except for $\rho=\tau \sigma^{-1}$. Hence,

$$
\left.\underline{F}_{\lambda, E}(x \sigma)=\sum_{n<N_{\tau \sigma^{-1}}} h_{n}^{\tau \sigma^{-1}}(x) \Pi_{\lambda}^{-1}(\sigma) \underline{\left(a_{\tau \sigma^{-1}, n, \lambda}\right.}\right) .
$$

On the other hand, $y=x \sigma \tau^{-1} \in D_{E} \sigma \tau^{-1}$, and $h_{n}^{\rho}(y \rho)=0$ except for $\rho=\tau \sigma^{-1}$. Thus,

$$
\begin{aligned}
\underline{F}_{\lambda, F}(y \tau) & =\sum_{n<N_{\tau \sigma^{-1}}} h_{n}^{\tau \sigma^{-1}}\left(y \tau \sigma^{-1}\right) \Pi_{\lambda}^{-1}(\tau) \underline{\left(b_{\tau \sigma^{-1}, n, \lambda}\right)} \\
& \left.=\sum_{n<N_{\tau \sigma^{-1}}} h_{n}^{\tau \sigma^{-1}}(x) \Pi_{\lambda}^{-1}(\tau) \Pi_{\lambda}\left(\tau \sigma^{-1}\right) \underline{\left(a_{\tau \sigma^{-1}, n, \lambda}\right.}\right)=\underline{F}_{\lambda, E}(x \sigma) .
\end{aligned}
$$

This demonstrates the independence.

Step 5. Finally, we will observe that the map $U$ preserves every $T(g), g \in$ $\operatorname{Diff}_{0}(M)$. Take any $f \in \mathrm{L}_{\nu_{E}}^{2}\left(M^{\infty}\right)$ and let

$$
U(T(g) f):=\int_{-} \underline{K}_{\lambda} \sqrt{d \sigma(\lambda)}
$$


Then, take $g D_{E}$ as $D_{F}$ and take a c.o.n.s. $\left\{T(g) h_{n}\right\}_{n}$ of $\mathrm{L}_{\nu_{E}}^{2}\left(g D_{E}\right)$, where $\left\{h_{n}\right\}_{n}$ is a c.o.n.s. of $\mathrm{L}_{\nu_{E}}^{2}\left(D_{E}\right)$. Then, for all $\sigma \in \mathfrak{S}_{\infty}$,

$$
\int_{g D_{E}}(T(g) f)(x \sigma) \overline{\left(T(g) h_{n}\right)(x)} \nu_{E}(d x)=\int_{D_{E}} f(x \sigma) \overline{h_{n}(x)} \nu_{E}(d x)=: a_{n}(\sigma) .
$$

Thus, for any $y \in g D_{E}$ and $\sigma \in \mathfrak{S}_{\infty}$,

$$
\begin{aligned}
\underline{K}_{\lambda}(y \sigma) & =\sum_{n}\left(T(g) h_{n}\right)(y) \Pi_{\lambda}^{-1}(\sigma) \underline{\left(a_{n, \lambda}\right)} \\
& =\sqrt{\frac{d g \nu_{E}}{d \nu_{E}}}(y) \sum_{n} h_{n}\left(g^{-1} y\right) \Pi_{\lambda}^{-1}(\sigma) \underline{\left(a_{n, \lambda}\right)} \\
& =\sqrt{\frac{d g \nu_{E}}{d \nu_{E}}}(y \sigma) \sum_{n} h_{n}\left(g^{-1} y\right) \Pi_{\lambda}^{-1}(\sigma) \underline{\left(a_{n, \lambda}\right)} \\
& =\sqrt{\frac{d g \nu_{E}}{d \nu_{E}}}(y \sigma) \underline{F}_{\lambda}\left(g^{-1} y \sigma\right)=\left(T(g) \underline{F}_{\lambda}\right)(y \sigma) .
\end{aligned}
$$

This shows that

$$
U(T(g) f)=\int_{-} T(g) \underline{F}_{\lambda} \sqrt{d \sigma(\lambda)} .
$$

Since $\left(T(g), \mathcal{H}\left(\Sigma_{\lambda}\right)\right), g \in \operatorname{Diff}_{0}(M)$, is irreducible, an irreducible decomposition of the natural representation $\left(T(g), \mathrm{L}_{\nu_{E}}^{2}\left(M^{\infty}\right)\right), g \in \operatorname{Diff}_{0}(M)$, is obtained.

\section{Appendix}

\section{$\S A .1$. Irreducibility}

We begin this section with the restatement and proof of Theorem 2.3.

Theorem 2.3 (irreducibility). Let $E$ be a disjoint $\mu$-unital sequence, and $(\Pi, H)$ an irreducible unitary representation of $\mathfrak{S}_{\infty}$. Form a triplet $\Sigma=(\mu, E, \Pi)$, as before. If $\operatorname{dim}(M) \geq 3$ and $M$ satisfies condition (mcc), then the unitary representation $(T, \mathcal{H}(\Sigma))$ is irreducible.

Proof. The proof consists of several steps. First we need the following lemma, which was already used in Section 3, and whose proof runs parallel to arguments in the finite-dimensional case.

Lemma A. Let $G=\left\{G_{n}\right\}_{n}$ be a disjoint $\mu$-unital sequence, and assume that each $G_{n}$ is connected and open. Then the natural representation $T$ of the restricted product group $\prod_{n}^{*} \operatorname{Diff}_{0}\left(G_{n}\right)$ on $\mathrm{L}_{\nu_{G}}^{2}(G)$, defined below, is irreducible:

$$
T(g): f(x) \mapsto \sqrt{\frac{d g \nu_{G}}{d \nu_{G}}}(x) f\left(g^{-1} x\right) .
$$


Next using the same reasonings as above, assume that $M$ satisfies condition (cc) and $E=\left\{E_{n}\right\}_{n}$ has the properties in Lemma 2.1. Put

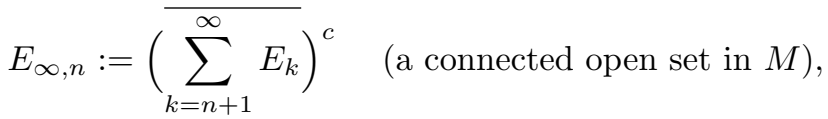

$$
\begin{aligned}
& \tilde{E}_{n}:=\bigcup_{\sigma \in \mathfrak{S}_{\infty}}\left(\left(E_{\infty, n}\right)^{n} \times \prod_{n+1}^{\infty} E_{k}\right) \sigma \quad\left(\text { a symmetric set in } M^{\infty}\right), \\
& P_{n}: f \in \mathcal{H}(\Sigma) \mapsto \chi_{\tilde{E}_{n}} \cdot f \in \mathcal{H}(\Sigma) \quad \text { (an increasing projection tending to id). }
\end{aligned}
$$

In addition, take any non-empty disjoint connected open sets $G_{1}, \ldots, G_{n}$ in $E_{\infty, n}$ such that for all $i, 0<\mu\left(G_{i}\right)<\infty$, form a unital sequence

$$
G E:=\left\{G_{1}, \ldots, G_{n}, E_{n+1}, \ldots, E_{k}, \ldots\right\}
$$

and define a map $Q_{G E}^{\Pi}$ by

$$
Q_{G E}^{\Pi}: f(x) \in \mathrm{L}_{\nu_{E}}^{2}\left(\prod_{k=1}^{n} G_{k} \times \prod_{k=n+1}^{\infty} E_{k}, H\right) \mapsto \sum_{\sigma \in \mathfrak{S}_{\infty}} \Pi(\sigma) f(x \sigma) \in \mathcal{H}(\Sigma) .
$$

Now let $A$ be an intertwining operator of $(T, \mathcal{H}(\Sigma))$. Then, after defining maps similar to $Q_{G E}^{\Pi}$, for example

$$
f(x) \in \mathrm{L}_{\nu_{E}}^{2}\left(\prod_{k=1}^{n} G_{i_{k}} \times \prod_{k=n+1}^{\infty} E_{k}, H\right) \mapsto \sum_{\sigma \in \mathfrak{S}_{\infty}} \Pi(\sigma) f(x \sigma) \in \mathcal{H}(\Sigma),
$$

where $i_{k}=1, \ldots, n$ or $=\infty$ with $G_{\infty}:=E_{\infty, n} \backslash \bigcup_{i=1}^{n} G_{i}$, and after some additional arguments, we get

$$
\operatorname{Image}\left(P_{n} A Q_{G E}^{\Pi}\right) \subset \operatorname{Image}\left(Q_{G E}^{\Pi}\right),
$$

in view of Lemma A. It follows that

$$
P_{n} A Q_{G E}^{\Pi}=Q_{G E}^{\Pi}\left(\mathrm{id}_{\mathrm{L}_{\nu_{E}}^{2}} \otimes A_{n, G}\right),
$$

where $A_{n, G}$ is a bounded operator on $H$, due to the same reason and the irreducibility assumption on $(\Pi, H)$.

As $A_{n, G}$ does not depend on the choice of $\left(G_{1}, \ldots, G_{n}\right)$ in view of the connectedness of $E_{\infty, n}$, we may simply write $A_{n}$ instead of $A_{n, G}$.

Now after some calculations using standard arguments of representation theory, we have

$$
\forall \sigma \in \mathfrak{S}_{n}, \quad \Pi(\sigma) A_{n}=A_{n} \Pi(\sigma), \quad \text { and } \quad \forall n, \quad A_{n}=A_{n+1}\left(=: A_{\infty}\right) .
$$

Thus, we see that there exists $c \in \mathbb{C}$ such that $A_{\infty}=c \mathrm{Id}$, because of the irreducibility of $(\Pi, H)$. 
In other words,

$$
P_{n} A Q_{G E}^{\Pi}=c Q_{G E}^{\Pi} \quad \text { and } \quad P_{n} A P_{n}=c P_{n},
$$

where the first equality follows from the fact that the family of subspaces $\left\{\operatorname{Image}\left(Q_{G E}^{\Pi}\right)\right\}_{G}, G:=\left(G_{1}, \ldots, G_{n}\right)$, generates the space $P_{n}(\mathcal{H}(\Sigma))$, whenever $G$ runs through all possible pairs of sets in $\left(E_{\infty, n}\right)^{n}$.

Finally, letting $n \rightarrow \infty$ in (5.1), we conclude that $A$ is a scalar operator $c$ Id. This gives also another proof of Theorem 2.3, though we have omitted the details.

\section{$\S$ A.2. Equivalence}

The rest of this section is devoted to a study of the mutual equivalence of $(T, \mathcal{H}(\Sigma))$, when $E$ runs through $\mu$-unital sets.

Theorem A. Assume that $\operatorname{dim}(M) \geq 3$ and let $M$ satisfy condition (mcc). Given $\Sigma_{1}=\left(\mu, E, \Pi_{1}\right)$ and $\Sigma_{2}=\left(\mu, F, \Pi_{2}\right),\left(T, \mathcal{H}\left(\Sigma_{1}\right)\right)$ and $\left(T, \mathcal{H}\left(\Sigma_{2}\right)\right)$ are unitarily equivalent if and only if

(1) there exists a permutation a on $\mathbb{N}$ (maybe infinite) such that $E \sim F a^{-1}$,

(2) $\Pi_{1}$ is equivalent to ${ }^{a} \Pi_{2}$ defined by

$$
{ }^{a} \Pi_{2}(\sigma):=\Pi_{2}\left(a^{-1} \sigma a\right) \quad \text { for } \sigma \in \mathfrak{S}_{\infty} .
$$

Proof. The sufficiency is obvious. The difficulty lies in showing the necessity, in particular, that there exists an infinite permutation $\sigma$ on $\mathbb{N}$ such that $\sum_{n=1}^{\infty} \mu\left(E_{n} \ominus F_{\sigma(n)}\right)<\infty$ (Lemma G). To do this, we first prepare a useful lemma (Lemma B), which has many applications as an ordinary check of the irreducibility of natural representations of the normal type. Applying the lemma, we find that $E_{n}$ overlaps widely with a unique $F_{\sigma(n)}$ for sufficiently large $n$. That is, $\lim _{n \rightarrow \infty} \mu\left(E_{n} \ominus F_{\sigma(n)}\right)=0$ (Lemma C). To complete the proof, we need to analyze $M$ further, and this requires another lemma (Lemma D) that states the possibility of incompressive transportation of mass from one part to another of an open, connected set in $M$. The rest of the proof discusses how to use this transportation lemma, and requires a lengthy technical argument. Finally, we claim that $\sigma$ is actually a permutation of $\mathbb{N}$. This is an outline of our original proof. Note that we may assume that $M$ satisfies (cc) as before.

Lemma B. Let $M$ satisfy (cc), suppose that $\left(T, \mathcal{H}\left(\Sigma_{1}\right)\right)$ and $\left(T, \mathcal{H}\left(\Sigma_{2}\right)\right)$ are unitarily equivalent and let $A$ be an intertwining unitary operator, $A: \mathcal{H}\left(\Sigma_{1}\right) \rightarrow$ $\mathcal{H}\left(\Sigma_{2}\right)$. Given a Borel set $B \in \mathfrak{B}(M)$, we introduce a projection $P_{B}$ on $\mathcal{H}\left(\Sigma_{i}\right)$ 
$(i=1,2)$ by

$$
\left(P_{B} f\right)(x):=\prod_{n=1}^{\infty} \chi_{B}\left(x_{n}\right) f(x)
$$

Then

$$
A P_{B}=P_{B} A
$$

Proof. Without loss of generality we may, of course, assume that $E=\left\{E_{n}\right\}_{n}$ and $F=\left\{F_{n}\right\}_{n}$ have the properties in Lemma 2.1.

Step 0 (Preparation). Suppose that $D$ a given relatively compact, open set. Given $\eta_{1}$, take a compact subset $K$ of $D$ such that $\mu(D \backslash K)<\eta_{1}$ and cover $K$ with a finite collection $\left\{W_{t}\right\}_{t=1}^{T}$ of relatively compact, open sets diffeomorphic to disks in $\mathbb{R}^{d}$ :

$$
K \subset \bigcup_{t=1}^{T} W_{t} \subseteq D
$$

Without loss of generality, we may assume that the image of $\mu \mid W_{t}$ under the coordinate map $\phi_{t}: W_{t} \rightarrow \mathbb{R}^{d}$ is the restriction of the Lebesgue measure to $\phi_{t}\left(W_{t}\right)$ and that $\mu\left(\bar{W}_{t} \backslash W_{t}\right)=0$. Put

$$
V_{1}:=W_{1}, \quad V_{t}:=W_{t} \backslash\left(\bar{W}_{1} \cup \cdots \cup \bar{W}_{t-1}\right) \quad(t=2, \ldots, T) .
$$

Then $V_{t}(t=1, \ldots, T)$ are mutually disjoint, open sets,

$$
\mu\left(\bigcup_{t=1}^{T} W_{t} \backslash \bigcup_{t=1}^{T} V_{t}\right)=0, \quad \text { and hence } \mu\left(K \backslash \bigcup_{t=1}^{T} V_{t}\right)=0 .
$$

Given $\eta_{2}>0$, take an open set $U_{t}$ such that

$$
\bar{U}_{t} \subset V_{t} \quad \text { and } \quad \mu\left(V_{t} \backslash U_{t}\right)<\eta_{2} \quad(t=1, \ldots, T) .
$$

Moreover, for $0<a<1$ and each $t$, take $\tilde{g}_{t}^{a, \eta} \in \operatorname{Diff}_{0}\left(\phi_{t}\left(W_{t}\right)\right)$ such that

$$
\left(\tilde{g}_{t}^{a, \eta}\right)^{-1}(x)=a x \quad \text { on } \phi_{t}\left(\bar{U}_{t}\right) .
$$

Finally, put

$$
g_{t}^{a, \eta}:=\phi_{t}^{-1} \circ \tilde{g}_{t}^{a, \eta} \circ \phi_{t} \quad \text { and } \quad g^{a, \eta}:=\prod_{t=1}^{T} g_{t}^{a, \eta} .
$$

Then $g^{a, \eta} \in \operatorname{Diff}_{0}(D)$ and

$$
\int_{D} \sqrt{\frac{d g^{a, \eta} \mu}{d \mu}}(P) \mu(d P) \leq a^{d / 2} \mu\left(\bigcup_{t=1}^{T} U_{t}\right)+\sqrt{\mu(D)} \sqrt{\mu\left(D \backslash \bigcup_{t=1}^{T} U_{t}\right)} .
$$


Hence, letting first $a \rightarrow+0$ and then $\eta_{1}, \eta_{2} \rightarrow+0$, we get

$$
\lim _{a, \eta \rightarrow+0} \int_{D} \sqrt{\frac{d g^{a, \eta} \mu}{d \mu}}(P) \mu(d P)=0 .
$$

Step 1. In this step, we will prove the assertion for the case when $B$ is the complement of a relatively compact, open set $D$.

To this end, we need to check that

$$
\left\langle P_{D^{c}} A \phi, A \phi\right\rangle_{\mathcal{H}\left(\Sigma_{2}\right)}=\left\langle P_{D^{c}} \phi, \phi\right\rangle_{\mathcal{H}\left(\Sigma_{1}\right)} \quad \text { for all } \phi \in \mathcal{H}\left(\Sigma_{1}\right)
$$

and this is ensured if we show that

$$
\left\langle T\left(g^{a, \eta}\right) \phi, \phi\right\rangle_{\mathcal{H}\left(\Sigma_{i}\right)} \rightarrow\left\langle P_{D^{c}} \phi, \phi\right\rangle_{\mathcal{H}\left(\Sigma_{i}\right)}
$$

as $a, \eta \rightarrow+0$. Since the same proof works for $i=1,2$, we have only to check it for $i=1$. Moreover, as can be easily seen, we may assume that $\|\phi\|_{H_{1}}$ is bounded $\left(\|\phi\|_{\infty}<\infty\right)$, as we prove (5.3).

Let $p_{k}$ denote the natural $k$ th projection from $M^{\infty}$ to $M$, and $M_{E}^{(n)}:=M^{n} \times$ $\prod_{k=n+1}^{\infty} E_{k}$, and set

$$
\begin{aligned}
L_{k}^{a, \eta} & :=\int_{D_{E} \cap p_{k}^{-1}(D)} \sqrt{\frac{d g^{a, \eta} \nu_{E}}{d \nu_{E}}}(x)\left|\left\langle\phi\left(\left(g^{a, \eta}\right)^{-1}(x)\right), \phi(x)\right\rangle_{H_{1}}\right| \nu_{E}(d x) \\
& =L_{k, n}^{a, \eta, 1}+L_{k, n}^{a, \eta, 2}
\end{aligned}
$$

where

$$
L_{k, n}^{a, \eta, 1}:=\int_{D_{E} \cap p_{k}^{-1}(D) \cap M_{E}^{(n)}}(\ldots), \quad L_{k, n}^{a, \eta, 2}:=\int_{D_{E} \cap p_{k}^{-1}(D) \cap\left(M_{E}^{(n)}\right)^{c}}(\ldots) .
$$

It can be easily checked that

$$
L_{k, n}^{a, \eta, 2} \leq\|\phi\|_{\mathcal{H}\left(\Sigma_{1}\right)}\left\{\int_{D_{E} \cap\left(M_{E}^{(n)}\right)^{c}}\|\phi(x)\|_{H_{1}}^{2} \nu_{E}(d x)\right\}^{1 / 2}
$$

hence it converges to 0 uniformly in $a, \eta$ as $n \rightarrow \infty$.

Take a sequence $\left\{K_{s}\right\}_{s \in \mathbb{N}}$ of compact sets such that $K_{s} \uparrow M(s \rightarrow \infty)$, and put

$$
M_{E}^{(n, s)}:=\left(K_{s}\right)^{n} \times \prod_{k=n+1}^{\infty} E_{k}
$$

and

$$
L_{k, n, s}^{a, \eta, 1}:=\int_{D_{E} \cap p_{k}^{-1}(D) \cap M_{E}^{(n, s)}} \sqrt{\frac{d g^{a, \eta} \nu_{E}}{d \nu_{E}}}(x)\left|\left\langle\phi\left(\left(g^{a, \eta}\right)^{-1}(x)\right), \phi(x)\right\rangle_{H_{1}}\right| \nu_{E}(d x) .
$$


Then we get

$$
\left|L_{k, n}^{a, \eta, 1}-L_{k, n, s}^{a, \eta, 1}\right| \leq\|\phi\|_{\mathcal{H}\left(\Sigma_{1}\right)}\left\{\int_{D_{E} \cap\left(M_{E}^{(n)} \backslash M_{E}^{(n, s)}\right)}\|\phi(x)\|_{H_{1}}^{2} \nu_{E}(d x)\right\}^{1 / 2}
$$

which converges to 0 uniformly in $(a, \eta)$ for each fixed $n$ as $s \rightarrow \infty$. Moreover, after some calculations, we get

$$
\begin{aligned}
L_{k, n, s}^{a, \eta, 1} & \leq\|\phi\|_{\infty}^{2} \int_{M_{E}^{(n, s)} \cap p_{k}^{-1}(D)} \sqrt{\frac{d g^{a, \eta} \nu_{E}}{d \nu_{E}}}(x) \nu_{E}(d x) \\
& \leq\|\phi\|_{\infty}^{2}\left(\mu\left(K_{s}\right)\right)^{n-1} \prod_{k=n+1}^{\infty} \mu\left(E_{k}\right) \int_{D} \sqrt{\frac{d g^{a, \eta} \mu}{d \mu}}(P) \mu(d P)
\end{aligned}
$$

provided that $k<n$ and $s$ and $n$ are so large that

$$
D \subset K_{s} \quad \text { and } \quad E_{k} \cap D=\emptyset \quad \text { for all } k \geq n+1 .
$$

It follows from (5.2), (5.5) and (5.6) that

$$
\lim _{a, \eta \rightarrow+0} L_{k}^{a, \eta}=0 \quad \text { for all } k \in \mathbb{N} .
$$

Obviously, we have

$$
\begin{aligned}
& \left\langle T\left(g^{a, \eta}\right) \phi, \phi\right\rangle_{\mathcal{H}\left(\Sigma_{1}\right)} \\
& =\int_{D_{E} \cap \bigcup_{k} p_{k}^{-1}(D)} \sqrt{\frac{d g^{a, \eta} \nu_{E}}{d \nu_{E}}}(x)\left\langle\phi\left(\left(g^{a, \eta}\right)^{-1}(x)\right), \phi(x)\right\rangle_{H_{1}} \nu_{E}(d x) \\
& \quad+\int_{D_{E} \cap \bigcap_{k} p_{k}^{-1}\left(D^{c}\right)}\|\phi(x)\|_{H_{1}}^{2} \nu_{E}(d x),
\end{aligned}
$$

and the second term on the right-hand side is equal to $\left\langle P_{D^{c}} \phi, \phi\right\rangle_{\mathcal{H}\left(\Sigma_{1}\right)}$.

We take $n$ so large that $D \cap E_{k}=\emptyset$ for all $k \geq n+1$ and split the first term on the right-hand side in (5.8) as follows:

$$
\int_{D_{E} \cap \bigcup_{k} p_{k}^{-1}(D) \cap M_{E}^{(n)}}(\ldots)+\int_{D_{E} \cap \bigcup_{k} p_{k}^{-1}(D) \cap\left(M_{E}^{(n)}\right)^{c}}(\ldots) .
$$

The absolute value of the first integral is smaller than

$$
\sum_{k=1}^{n} \int_{D_{E} \cap p_{k}^{-1}(D)}|(\ldots)|
$$

and it converges to 0 as $a, \eta \rightarrow+0$ for fixed $n$. 
On the other hand, the absolute value of the second integral is smaller than

$$
\|\phi\|_{\mathcal{H}\left(\Sigma_{1}\right)}\left\{\int_{D_{E} \cap\left(M_{E}^{(n)}\right)^{c}}\|\phi(x)\|_{H_{1}}^{2} \nu_{E}(d x)\right\}^{1 / 2}
$$

which also converges to 0 as $n \rightarrow \infty$. It follows that the proof of $(5.4)$ is now complete.

Step 2. Suppose that $B$ is a closed set in $M$. Take a sequence $\left\{D_{n}\right\}_{n \in \mathbb{N}}$ of relatively compact, open sets such that $D_{n} \uparrow M$ and put $B_{n}^{c}:=D_{n} \cap B^{c}$. Then $B_{n} \downarrow B$, so that $P_{B_{n}} \downarrow P_{B}$. It follows from Step 1 that

$$
A P_{B_{n}}=P_{B_{n}} A, \text { and hence } A P_{B}=P_{B} A .
$$

Step 3. In particular, when $\mu(\bar{B} \backslash B)=0$, the above conclusion follows, because $P_{\bar{B}}=P_{B}$.

Step 4. Let us consider a general Borel set $B$. Put $B_{n}:=B \cup \bigcup_{k=n+1}^{\infty}\left(E_{k} \cup F_{k}\right)$. Then $B_{n} \downarrow B$, and hence

$$
P_{B_{n}} \downarrow P_{B}
$$

Further, take an increasing sequence $\left\{T_{m}\right\}_{m \in \mathbb{N}}$ of closed subsets of $B$ such that $\mu\left(B \backslash \bigcup_{m=1}^{\infty} T_{m}\right)=0$, and put $B_{n, m}:=T_{m} \cup \bigcup_{k=n+1}^{\infty}\left(E_{k} \cup F_{k}\right)$. Since $\mu\left(\overline{B_{n, m}} \backslash B_{n, m}\right)=0$, due to Lemma 2.1 , we get

$$
A P_{B_{n, m}}=P_{B_{n, m}} A
$$

by the step above. Now, put

$$
M_{E \cup F}^{(l)}:=M^{l} \times \prod_{l+1}^{\infty}\left(E_{k} \cup F_{k}\right) \quad \text { and } \quad B_{n}^{\prime}:=\bigcup_{m=1}^{\infty} T_{m} \cup \bigcup_{k=n+1}^{\infty}\left(E_{k} \cup F_{k}\right) .
$$

It can be easily seen that, for $n<l$,

$$
\left(B_{n, m} \times B_{n, m} \times \cdots\right) \cap M_{E \cup F}^{(l)} \uparrow\left(B_{n}^{\prime} \times B_{n}^{\prime} \times \cdots\right) \cap M_{E \cup F}^{(l)} \quad(m \rightarrow \infty) .
$$

Thus for fixed $n$,

$$
P_{B_{n, m}} \uparrow P_{B_{n}^{\prime}}=P_{B_{n}} \quad \text { on } \mathcal{H}\left(\Sigma_{i}\right)(i=1,2) .
$$

Hence, the conclusion follows from (5.9) and (5.10).

Lemma C. Under the same assumptions and the same notation as in Theorem A and assumption (cc) on $M$, the following holds: for sufficiently large $n \in \mathbb{N}$, there exists $\sigma(n) \in \mathbb{N}$ such that

$$
\lim _{n \rightarrow \infty} \mu\left(E_{n} \ominus F_{\sigma(n)}\right)=0
$$


Proof. As before, we may assume that $E=\left\{E_{n}\right\}_{n}$ and $F=\left\{F_{n}\right\}_{n}$ have the properties as in Lemma 2.1. Take $h \in H_{1}$ with $\|h\|_{H_{1}}=1$ and put

$$
g(x):=A Q_{E}^{\Pi_{1}}\left(\prod_{n=1}^{\infty} \chi_{E_{n}} \otimes h\right)(x) .
$$

Applying the above lemma to $B:=E_{k}^{c}$ for each $k$, we have

$$
P_{E_{k}^{c}} g=0 .
$$

Next, approximating $g$ with a sum of $Q_{F}^{\Pi_{2}}$-images of tame functions

$$
Q_{F}^{\Pi_{2}}\left(\rho\left(x_{1}, \ldots, x_{l}\right) \prod_{n=l+1}^{\infty} \chi_{F_{n}}\left(x_{n}\right) \cdot h^{\prime}\right)
$$

where $\rho$ is a square summable function and $h^{\prime} \in H_{2}$, we find that

$$
\forall \epsilon>0, \exists N_{\epsilon} \in \mathbb{N}, \forall k \geq N_{\epsilon}, \quad \prod_{n=1}^{\infty} \mu\left(E_{k}^{c} \cap F_{n}\right)<\epsilon .
$$

It follows that

$$
\exists \sigma(k) \in \mathbb{N}, \quad \mu\left(E_{k}^{c} \cap F_{\sigma(k)}\right)<\epsilon K_{U},
$$

with a universal constant $K_{U}$. By proceeding in a similar fashion, but changing $E$ to $F$, we can show that $\mu\left(E_{k} \cap F_{\sigma(k)}^{c}\right)$ is equally small if $k$ is large.

For the proof of Theorem A, we need more analysis on $M$. The following lemma is useful; it shows the possibility of incompressive transportation of mass from one part to another with slim tubes in connected open sets in $M$.

Lemma D. Assume that $d:=\operatorname{dim}(M) \geq 2$. Let $F$ be a connected, open subset of $M$ and $U_{i}(i=1,2)$ be open subsets of $F$ such that $U_{1} \cap U_{2}=\emptyset$ and $\mu\left(U_{1}\right)<$ $\mu\left(U_{2}\right)<\infty$. Then, given $\epsilon>0$, we have a $\mu$-preserving diffeomorphism $g_{\epsilon} \in$ $\operatorname{Diff}_{0}(F)$ and a Borel subset $B_{\epsilon} \subset U_{1}$ such that

$$
\mu\left(U_{1} \backslash B_{\epsilon}\right)<\epsilon \quad \text { and } \quad g_{\epsilon}\left(B_{\epsilon}\right) \subset U_{2} .
$$

Proof. In this proof, a $\lambda$-neighbourhood $V(P)$ of $P \in M$ is an open set including $x$ with the following properties:

(1) $V(P)$ is diffeomorphic to a disk in $\mathbb{R}^{d}$ under a coordinate map $\phi$,

(2) the image measure of $\mu \mid V(P)$ by $\phi$ is the restriction of the Lebesgue measure to $\phi(V(P))$. 
Now, take a $\lambda$-neighbourhood $V(P)\left(\subset U_{1}\right)$ for each $P \in U_{1}$ and cover $U_{1}$ by a countable collection of $\left\{V\left(P_{n}\right)\right\}_{n}$. Put

$$
W\left(P_{1}\right):=V\left(P_{1}\right) \quad \text { and } \quad W\left(P_{n}\right):=V\left(P_{n}\right) \backslash\left(\overline{V\left(P_{1}\right)} \cup \cdots \cup \overline{V\left(P_{n-1}\right)}\right) .
$$

Then $V\left(P_{n}\right) \cap W\left(P_{m}\right)=\emptyset(n \neq m)$ and

$$
\mu\left(U_{1} \backslash \sum_{n=1}^{N} W\left(P_{n}\right)\right)<\frac{1}{3} \epsilon \quad \text { for sufficiently large } N .
$$

Since $W\left(P_{n}\right)$ can be approximated as closely as we wish by finite unions of $\lambda$ neighbourhoods, in place of $W\left(P_{n}\right)$ we can take an open subset $\hat{W}\left(P_{n}\right)$ such that $\overline{\hat{W}\left(P_{n}\right)} \subset W\left(P_{n}\right), \hat{W}\left(P_{n}\right)$ is a finite union of inverse images of rectangles in $\mathbb{R}^{d}$ and

$$
\mu\left(\sum_{n=1}^{N} W\left(P_{n}\right) \backslash \sum_{n=1}^{N} \hat{W}\left(P_{n}\right)\right)<\frac{\epsilon}{3} .
$$

In exactly the same manner as for $U_{2}$, we have $V\left(Q_{m}\right), W\left(Q_{m}\right)$ and $\hat{W}\left(Q_{m}\right) \quad(m=1, \ldots, M)$ such that

$$
\begin{gathered}
\hat{W}\left(Q_{m}\right) \subset \overline{\hat{W}\left(Q_{m}\right)} \subset W\left(Q_{m}\right) \subset V\left(Q_{m}\right) \subset U_{2}, \\
\mu\left(U_{2} \backslash \sum_{m=1}^{M} \hat{W}\left(Q_{m}\right)\right)<\mu\left(U_{2}\right)-\mu\left(U_{1}\right) .
\end{gathered}
$$

Therefore,

$$
\mu\left(\sum_{n=1}^{N} \hat{W}\left(P_{n}\right)\right)<\mu\left(U_{1}\right)<\mu\left(\sum_{m=1}^{M} \hat{W}\left(Q_{m}\right)\right) .
$$

Now, we connect $V\left(P_{i}\right)$ and $V\left(P_{i+1}\right)(i=1, \ldots, N-1)$ by a slim open tube included in $F$ which intersects neither $\hat{W}\left(P_{n}\right)(n=1, \ldots, N)$ nor $\hat{W}\left(Q_{m}\right)(m=$ $1, \ldots, M)$. Similarly we proceed for $V\left(Q_{j}\right)$ and $V\left(Q_{j+1}\right)(j=1, \ldots M)$, and we finally connect $V\left(P_{1}\right)$ and $V\left(Q_{1}\right)$ by a slim open tube $S$ included in $F$ which intersects neither $\hat{W}\left(P_{n}\right)(n=1, \ldots, N)$ nor $\hat{W}\left(Q_{m}\right)(m=1, \ldots, M)$.

Now, we transport each divided small mass of $\hat{W}\left(P_{1}\right)$ to $\hat{W}\left(Q_{M}\right), \hat{W}\left(Q_{M-1}\right)$, $\ldots, \hat{W}\left(Q_{1}\right)$ through $S$ and through the slim tubes for $U_{2}$ (a larger subscript of $Q$ has priority in the order of distribution). In fact, this is possible by the following lemma.

Lemma E. There exists a Lebesgue measure preserving diffeomorphism with compact support that realizes local displacement in $\mathbb{R}^{d}(d \geq 2)$.

Proof. Put $n:=d-2$ and take compact intervals

$$
\left[\alpha^{\prime}, \beta^{\prime}\right] \subset[\alpha, \beta], \quad\left[\gamma^{\prime}, \delta^{\prime}\right] \subset[\gamma, \delta], \quad\left[\alpha_{i}^{\prime}, \beta_{i}^{\prime}\right] \subset\left[\alpha_{i}, \beta_{i}\right] \quad(i=1, \ldots, n) .
$$


We take a vector field $v \equiv\left(v_{1}, v_{2}, w_{1}, \ldots, w_{n}\right)$ on $\mathbb{R}^{d}$ defined by

$$
\begin{aligned}
v_{1}\left(x, y, z_{1}, \ldots, z_{n}\right) & :=f_{1}(x) f_{2}^{\prime}(y) g_{1}\left(z_{1}\right) \cdots g_{n}\left(z_{n}\right), \\
v_{2}\left(x, y, z_{1}, \ldots, z_{n}\right) & :=-f_{1}^{\prime}(x) f_{2}(y) g_{1}\left(z_{1}\right) \cdots g_{n}\left(z_{n}\right), \\
w_{i} & \equiv 0 \quad(i=1, \ldots, n)
\end{aligned}
$$

where $f_{1}, f_{2}$ and $g_{i}(i=1, \ldots, n)$ are $C^{\infty}$ functions on $\mathbb{R}^{1}$ with compact support such that

$$
f_{1}(x)=\left\{\begin{array}{ll}
1 & \text { on }\left[\alpha^{\prime}, \beta^{\prime}\right], \\
0 & \text { on }[\alpha, \beta]^{c},
\end{array} \quad f_{2}(y)=\left\{\begin{array}{ll}
y & \text { on }\left[\gamma^{\prime}, \delta^{\prime}\right], \\
0 & \text { on }[\gamma, \delta]^{c},
\end{array} \quad g_{i}(x)= \begin{cases}1 & \text { on }\left[\alpha_{i}^{\prime}, \beta_{i}^{\prime}\right] \\
0, & \text { on }\left[\alpha_{i}, \beta_{i}\right]^{c} .\end{cases}\right.\right.
$$

It is clear that

$$
\begin{gathered}
\operatorname{supp} v \subseteq[\alpha, \beta] \times[\gamma, \delta] \times \prod_{i=1}^{n}\left[\alpha_{i}, \beta_{i}\right], \quad \operatorname{div}(v)=0, \\
v=(1,0, \ldots, 0) \quad \text { on } T:=\left[\alpha^{\prime}, \beta^{\prime}\right] \times\left[\gamma^{\prime}, \delta^{\prime}\right] \times \prod_{i=1}^{n}\left[\alpha_{i}^{\prime}, \beta_{i}^{\prime}\right] .
\end{gathered}
$$

Therefore,

$$
\exp (t v)\left(x_{0}\right)=x_{0}+t(1,0, \ldots, 0) \quad \text { for all } x_{0} \in T,
$$

provided that $x_{0}+t(1,0, \ldots, 0) \in T$. This is the desired diffeomorphism.

The rest of the proof of Theorem A relies on the above lemma. We first give the following two lemmas; we omit their proofs, because they are quite technical and complicated (for details, see [18]).

Lemma F. Under the same assumptions and notation of Lemma $C$, we have

$$
\sum \max _{k \neq n} \mu\left(E_{k} \cap F_{\sigma(n)}\right)<\infty
$$

where the summation is over all $n$ except a finite number.

Lemma G. Under the same assumptions and notation of Lemma $C$, we have

$$
\sum \mu\left(E_{n} \ominus F_{\sigma(n)}\right)<\infty
$$

where the summation is over all $n$ except a finite number.

Now we can complete the proof of Theorem A through standard arguments of representation theory in order to see that $\sigma$ actually extends to a permutation on $\mathbb{N}$ and to show the equivalence of the irreducible unitary representations $(\Pi, H)$.

In a similar fashion, we get an interesting version of Theorem A. 
Theorem B. Assume that $\operatorname{dim}(M) \geq 3$, and let $M$ satisfy (mcc). Given $\mu, E$, $F, H_{1}, H_{2}$, we have a nonzero intertwining operator $A:\left(T, \mathrm{~L}_{\nu_{E}}^{2}\left(M^{\infty}, H_{1}\right)\right) \rightarrow$ $\left(T, \mathrm{~L}_{\nu_{F}}^{2}\left(M^{\infty}, H_{2}\right)\right)$ if and only if there exists a permutation a on $\mathbb{N}$ (maybe infinite) such that $E \sim F a^{-1}$.

\section{Acknowledgements}

I wish to express my deep gratitude to Honorary Professor T. Hirai of Kyoto University for introducing me to this subject. In fact, the underlying concepts are due to him.

This research was partially supported by a Grant-in-Aid for Scientific Research (No. 16540162) from the Japan Society of the Promotion of Science.

\section{References}

[1] G. A. Goldin, Nonrelativistic current algebras as unitary representations of groups, J. Math. Phys. 12 (1971), 462-487. MR 0284101

[2] G. A. Goldin, J. Grodnik, R. T. Powers, and D. H. Sharp, Nonrelativisitic current algebra in the $N / V$ limit, J. Math. Phys. 15 (1974), 88-100.

[3] T. Hirai, Construction of irreducible unitary representations of the infinite symmetric group $\mathfrak{S}_{\infty}$, J. Math. Kyoto Univ. 31 (1991), 495-541. Zbl 0752.22001 MR 1121176

[4] - Irreducible unitary representations of the group of diffeomorphisms of a noncompact manifold, J. Math. Kyoto Univ. 33 (1993), 827-864. Zbl 0813.58006 MR 1239096

[5] T. Hirai and H. Shimomura, Relations between unitary representations of diffeomorphism groups and those of the infinite symmetric group or of related permutation groups, J. Math. Kyoto Univ. 37 (1997), 261-316. Zbl 0897.22008 MR 1463849

[6] R. S. Ismagilov, Unitary representations of the group of diffeomorphisms of a circle, Funktsional. Anal. i Prilozhen. 5 (1971), no. 3, 45-53 (in Russian): English transl.: Funct. Anal. Appl. 5 (1971), 209-216. Zbl 0235.58006 MR 0285671

[7] $\_$Representations of infinite-dimensional groups, Transl. Math. Monogr. 152, Amer. Math. Soc. Appl. Providence, RI, 1996. Zbl 0856.22001 MR 1393939

[8] F. I. Mautner, Unitary representations of locally compact groups, I, Ann. of Math. 51 (1950), 1-25. Zbl 0035.29802 MR 0032650

[9] Unitary representations of locally compact groups, II, Ann. of Math. 52 (1950), 528-556. Zbl 0039.02201 MR 0036763

[10] C. C. Moore, Invariant measures on product spaces, in Proc. fifth Berkeley sympos. math. statist. and probability (Berkeley, CA, 1965/66), Vol. II: Contributions to probability theory, Part 2, Univ. California Press, Berkeley, CA, 1967, 447-459. Zbl 0208.06801 MR 0227366

[11] F. Murray and J. von Neumann, On rings of operators, Ann. of Math. 37 (1936), 116-229. Zbl 0014.16101 MR 1503275

[12] J. von Neumann, On infinite direct products, Compos. Math. 6 (1938), 1-77. JFM 64.0377.01 MR 1557013

[13] Rings of operators. Reduction theory, Ann. of Math. 50 (1949), 401-485. Zbl 0034.06102 MR 0029101

[14] H. Omori, Infinite-dimensional Lie groups, Transl. Math. Monogr. 158, Amer. Math. Soc., Providence, RI, 1997. Zbl 0871.58007 MR 1421572 
[15] E. Shavgulidze, Mesures quasi-invariantes sur les groupes de difféomorphismes des variétés riemaniennes, C. R. Acad. Sci. Paris 321 (1995), 229-232. Z Zbl 0841.58009 MR 1345453

[16] H. Shimomura, Poisson measures on the configuration space and unitary representations of the group of diffeomorphisms, J. Math. Kyoto Univ. 34 (1994), 599-614. Zbl 0874.57028 MR 1295944

[17] Q Quasi-invariant measures on the group of diffeomorphisms and smooth vectors of unitary representations, J. Funct. Anal. 187 (2001), 406-441. Zbl 0997.58004 MR 1875154

[18] Unitary representations of the group of diffeomorphisms via restricted product measures with infinite mass, in Infinite dimensional harmonic analysis III, World Sci., 2005, 289-311. Zbl 1117.22011 MR 2230637

[19] - Irreducible decompositions of unitary representations of infinite-dimensional groups, Math. Z. 251 (2005), 575-587. Zbl 1087.58006 MR 2190345

[20] A. M. Vershik, I. M. Gel'fand and M. I. Graev, Representations of the group of diffeomorphism, Uspekhi Mat. Nauk 30 (1975), no. 6, 1-50 (in Russian): English transl.: Russian Math. Surveys 30 (1975), no. 6, 3-50. Zbl 0337.58003

[21] Y. Yamasaki, Measures on infinite dimensional spaces, World Sci., 1985. Zbl 0591.28012 MR 0999137 